\title{
Neural plasticity expressed in central auditory structures with and without tinnitus
}

\author{
Larry E. Roberts*, Daniel J. Bosnyak and David C. Thompson
}

Department of Psychology, Neuroscience, and Behaviour, McMaster University, Hamilton, ON, Canada

\section{Edited by:}

Jos J. Eggermont, University of

Calagry, Canada

Reviewed by:

Eugen Diesch, Central Institute of Mental Health, Germany

Kelly Tremblay, University of

Washington, USA

*Correspondence:

Larry E. Roberts, Department of

Psychology, Neuroscience, and

Behaviour, McMaster University,

1280 Main Street West, Hamilton,

ON L8S 4K1, Canada.

e-mail: roberts@mcmaster.ca
Sensory training therapies for tinnitus are based on the assumption that, notwithstanding neural changes related to tinnitus, auditory training can alter the response properties of neurons in auditory pathways. To assess this assumption, we investigated whether brain changes induced by sensory training in tinnitus sufferers and measured by electroencephalography (EEG) are similar to those induced in age and hearing loss matched individuals without tinnitus trained on the same auditory task. Auditory training was given using a $5 \mathrm{kHz} 40-\mathrm{Hz}$ amplitude-modulated (AM) sound that was in the tinnitus frequency region of the tinnitus subjects and enabled extraction of the $40-\mathrm{Hz}$ auditory steady-state response (ASSR) and P2 transient response known to localize to primary and non-primary auditory cortex, respectively. P2 amplitude increased over training sessions equally in participants with tinnitus and in control subjects, suggesting normal remodeling of non-primary auditory regions in tinnitus. However, training-induced changes in the ASSR differed between the tinnitus and control groups. In controls the phase delay between the $40-\mathrm{Hz}$ response and stimulus waveforms reduced by about $10^{\circ}$ over training, in agreement with previous results obtained in young normal hearing individuals. However, ASSR phase did not change significantly with training in the tinnitus group, although some participants showed phase shifts resembling controls. On the other hand, ASSR amplitude increased with training in the tinnitus group, whereas in controls this response (which is difficult to remodel in young normal hearing subjects) did not change with training. These results suggest that neural changes related to tinnitus altered how neural plasticity was expressed in the region of primary but not non-primary auditory cortex. Auditory training did not reduce tinnitus loudness although a small effect on the tinnitus spectrum was detected.

Keywords: tinnitus, neural plasticity, auditory system, sensory training, EEG

\section{INTRODUCTION}

Tinnitus is a phantom sound (ringing of the ears) that affects quality of life for millions around the world and is a major challenge for health systems because effective medical treatments are lacking. Most cases are associated with hearing impairment detected by the audiogram (Henry and Meikle, 2000) or more sensitive measures (Weisz et al., 2006; Kujawa and Liberman, 2009; Schaette and McAlpine, 2011). One of the neural changes consequent on hearing loss is tonotopic map reorganization in which neurons in the hearing loss region of primary auditory cortex (A1) begin to express the tuning preferences of their unaffected neighbors, thereby augmenting the representation of neighboring frequencies in the cortical place map (Rajan and Irvine, 1998; Noreña et al., 2003). Map reorganization, which has been documented in human tinnitus sufferers with hearing loss (Wienbruch et al., 2006), suggests that pre-existing inputs on lateral connections to neurons in the hearing loss region now have a stronger influence on these neurons than do surviving inputs from thalamocortical pathways (Eggermont and Roberts, 2004). Other hearing loss-induced changes include shifts in the balance of excitation and inhibition in auditory cortical networks (Scholl and Wehr, 2008), increased spontaneous activity of neurons in central auditory structures (Noreña and Eggermont, 2003; Kaltenbach et al., 2004), increased burst firing in some of these structures (Finlayson and Kaltenbach, 2009), and increased synchronous activity among cortical neurons affected by hearing loss (Seki and Eggermont, 2003). Although the contribution of these changes to tinnitus percepts is not fully understood, enhanced neural synchrony is a likely proximal neural correlate because it is largely confined to the hearing loss frequencies (Noreña and Eggermont, 2003) where in human subjects tinnitus percepts also localize (Noreña et al., 2002; Roberts et al., 2008).

Forms of neural plasticity are believed to contribute to these neural changes following hearing impairment. Cochlear damage in an animal model of hearing loss is followed within two weeks by an upregulation of somatosensory inputs to auditory neurons in the dorsal cochlear nucleus (DCN), one of the early processing stages in subcortical auditory pathways (Zeng et al., 2009). This change is believed to reflect compensatory homeostatic plasticity that acts to preserve the global firing rates of deafferented auditory neurons within a prescribed dynamic range (Turrigiano and Nelson, 2004; Pozo and Goda, 2010). Homeostatic plasticity may explain why wave I of the auditory brain stem response (ABR) is reduced in tinnitus sufferers with normal audiograms, 
implying diminished auditory nerve activity consequent undetected cochlear damage, but wave $\mathrm{V}$ is not reduced (Schaette and McAlpine, 2011) suggesting compensatory changes in intervening central auditory structures. At the cortical level an undesirable consequence of compensatory change may be an increase in the spontaneous and driven activity of auditory neurons setting the stage for the development of tinnitus and hyperacusis which is reported by many tinnitus sufferers (Noreña, 2011). Increased neural synchrony is a further neural correlate of tinnitus that may result from neuroplastic mechanisms (Seki and Eggermont, 2003; Weisz et al., 2007). Following hearing loss and diminished feedforward inhibition, cortical neurons are likely to discharge in phase-locked patterns mediated by their lateral connections. Subsequently such activity may be forged into stable and larger functional assemblies by spike-timing dependent plasticity in the hearing loss region (cf. Yao and Dan, 2001), giving rise to persistent tinnitus sounds. These examples represent possible maladaptive consequences of neural plasticity operating in auditory regions affected by hearing loss. However, it has been proposed that therapeutic sensory training regimens may prevent or reverse maladaptive remodeling after hearing loss. In support of this hypothesis, prolonged exposure to low-level, complex high frequency sounds covering the region of threshold $(\mathrm{TH})$ shift prevents cortical map reorganization in cats subjected to noise trauma (Noreña and Eggermont, 2005) and has been reported to rescale loudness growth in human hyperacusis patients (Noreña and Chery-Croze, 2007). Improvements in peripheral hearing consequent on therapeutic sound appear to contribute to map preservation after noise trauma (Noreña and Eggermont, 2005), but rescaling of loudness growth in hyperacusis patients appears to depend on central adaptive mechanisms (Formby et al., 2003). Effects of therapeutic sound have been more variable applied to tinnitus percepts. Distress behavior associated with tinnitus is typically reduced following sensory training therapies, but effects on psychoacoustic measurements of tinnitus are inconsistent and have not been strongly realized (see Roberts and Bosnyak, 2010a; Hoare et al., 2011 for reviews).

Sensory training therapies are based on the assumption that the response properties of auditory neurons can be manipulated by neuroplastic mechanisms in the tinnitus brain, as is the case in normal hearing individuals. However, it is also possible that neural changes underlying tinnitus (for example, spontaneous or synchronous activity propagated by intact centrifugal pathways) may impede cortical remodeling in tinnitus sufferers. To address this question, we investigated whether brain changes induced by sensory training in individuals with tinnitus and measured by the electroencephalogram (EEG) are similar to those induced in age and hearing level matched individuals without tinnitus trained on the same auditory task. Training was given for a sound that was amplitude-modulated (AM) at $40 \mathrm{~Hz}$, which enabled extraction from the EEG of (1) the $40 \mathrm{~Hz}$ auditory steady-state response (ASSR) generated by cortical sources in posterior-medial Heschl's gyrus (A1; Brugge et al., 2009), and (2) the P2 transient response, which is believed to arise from distributed sources centered in non-primary (A2) auditory cortex (Picton et al., 1999). We thus gained a picture of changes occurring in both cortical regions from the same data-set (Bosnyak et al., 2004; Gander et al., 2010b). In previous research ASSR phase (the time delay between zero-crossings in the stimulus and response waveforms) and P2 amplitude have been found to be highly plastic revealing, respectively, changes in temporal population activity in A1 (Bosnyak et al., 2004; Gander et al., 2010b) and expansion of auditory representations in the region A2 (Tremblay et al., 2001; Reinke et al., 2003; Bosnyak et al., 2004; Sheehan et al., 2005; Ross and Tremblay, 2009; Gander et al., 2010b). In the current study auditory training was given for a sound in the tinnitus frequency region, to ensure that tinnitus networks were engaged in tinnitus subjects and to assess whether such training modified the tinnitus percept itself. Our goal was to determine whether tinnitus-related neural activity would affect the expression of plasticity in individuals with tinnitus compared to controls, and if neural plasticity was demonstrated in the tinnitus group, whether the tinnitus percept would be modulated.

Participants with chronic tinnitus $(n=11)$ and age and hearing-level matched controls $(n=11)$ participated in seven sessions of auditory training each separated by 1-3 days. Audiometric THs were measured for all participants in an initial session one week before auditory training commenced. Measurements of tinnitus spectra and loudness by the Tinnitus Tester method of Roberts et al. (2008) and tinnitus distress by the Tinnitus Handicap Questionnaire (THQ; Kuk et al., 1990) were taken in this session and repeated in a follow-up session one week after auditory training had ended. The stimulus for auditory training was a $5 \mathrm{kHz}$ pure tone of $976 \mathrm{~ms}$ duration AM with a $40.96 \mathrm{~Hz}$ sinusoid (called $40 \mathrm{~Hz}$ herein, $100 \%$ modulation depth). This carrier frequency was chosen because it is typically judged by tinnitus subjects to be in the tinnitus spectrum (Roberts et al., 2008; see Figure 2B). Sound level was matched by all participants to a $2 \mathrm{kHz} 40-\mathrm{Hz}$ AM reference tone presented at $65 \mathrm{~dB}$ SPL used in prior research (Gander et al., 2010b), so that findings could be contrasted across studies with perceived loudness controlled. The training procedure was identical to that of Gander et al. (2010b) except for the change in carrier frequency. During training (see Figure 1A) auditory stimuli were separated by an intertrial interval (ITI) of approximately $1900 \mathrm{~ms}$ during which behavioral responses were recorded. Approximately $2 / 3$ of the stimuli contained a single $40-\mathrm{Hz}$ AM pulse of variably increased amplitude (bracketing the $\mathrm{TH}$ of detection) that occurred randomly at times commencing $415 \mathrm{~ms}$ after stimulus onset (target). On active blocks participants pressed one of two mouse buttons after each trial indicating whether a target had or had not occurred (feedback for correctness was given). On passive blocks subjects ceased behavioral responding and ignored the auditory cues. Active and passive blocks alternated in sessions 1, 4, and 7 of auditory training during which the EEG was recorded. The remaining sessions were identical except that EEG was not recorded and all blocks were now active blocks. In all sessions, participants in the tinnitus group indicated using a slider their tinnitus "awareness" and "loudness" on a Borg CR100 scale (Borg and Borg, 2001) immediately before, at the midpoint, and the end of training (Figure 1B). ASSR amplitude and phase were extracted from the unmodeled data recorded by 128 EEG sensors using the method shown in Figure 1C (for further details see the Materials and Methods 


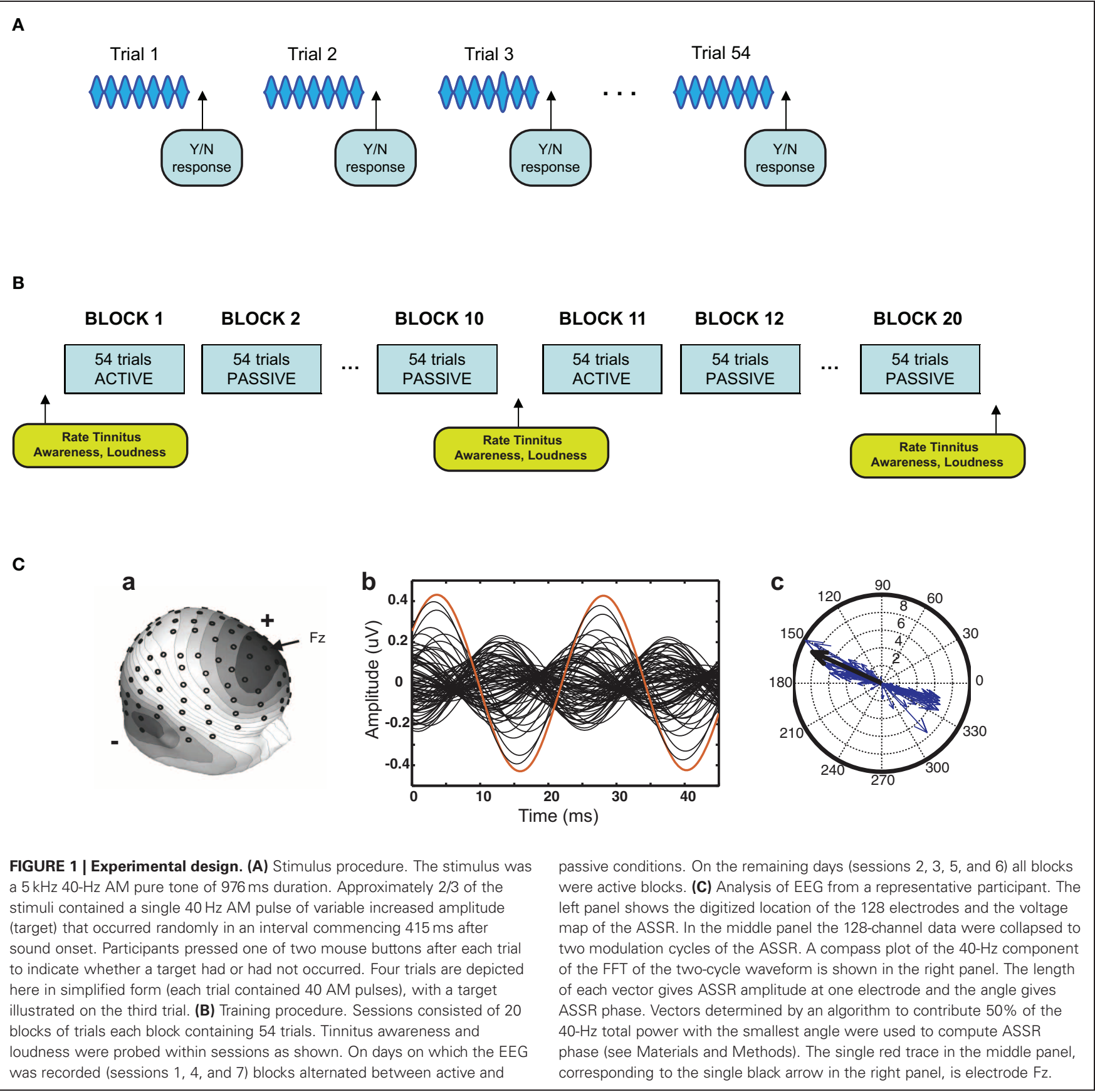

section). P2 and other transient responses were extracted by low pass filtering of the continuous EEG file and recorded at their amplitude maxima. In previous research we found that results obtained with these methods based on the unmodeled data concurred closely with results obtained when the cortical sources of ASSR and P2 responses were modeled in source space (Gander et al., 2010a,b).

\section{RESULTS}

Table 1 reports the mean age and age range of the tinnitus and control groups, their sound THs at 2 and $5 \mathrm{kHz}$ determined by clinical audiometry, and the sound level of the training stimulus delivered to each group. No significant group differences were found on any of these measures (all $p$ 's $>0.37$ ). Both groups experienced low to moderate hearing loss between 3 and $6 \mathrm{kHz}$ and deeper losses above this range (see Figure 2A), but no group differences were found at any frequency (main effect of frequency and group by frequency interaction $F$ 's $<1$ ). On average participants reported chronic tinnitus 11.7 years duration. All were bilateral cases with the majority reporting a narrow band "tonal" tinnitus and the remainder a tinnitus of greater bandwidth ("ringing" or "hissing") when comparing their tinnitus to 
Table 1 | Group information.

\section{Tinnitus Group Control Group}

\section{CHARACTERISTICS OF PARTICIPANTS}

Number (male)

$11(7)$

Age in Years Mean (SE)

$48.6(4.75)$

$11(6)$

Age Range in Years

22-68

$53.9(5.86)$

\section{AUDIOMETRIC DATA AND SOUND LEVELS}

Mean (SE) threshold @ 2 kHz Left

$10.0(4.54)$

$15.9(4.54)$

Ear (dB HL)

Mean (SE) threshold @ 2 kHz Right

$9.6(2.77)$

$7.7(2.77)$

Ear (dB HL)

Mean (SE) threshold @ 5 kHz Left

$29.4(7.65)$

$28.4(7.65)$

Ear (dB HL)

Mean (SE) threshold @ 5 kHz Right

$27.3(5.68)$

$23.0(5.68)$

Ear (dB HL)

Mean (SE) Training stimulus

$60.0(2.05)$

$58.5(2.01)$

loudness (dB SPL)a

\section{TINNITUS CHARACTERISTICS}

\section{Mean (SE) duration in years}

$11.7(3.03)$

Mean (SE) loudness rating Borg

$57.1(6.21)$

CR100 Scale S $^{\text {b }}$

Mean (SE) loudness match (1 kHz 53.9 (6.32)

tone, dB SPL) $)^{b}$

Tinnitus Bandwidth (number of

participants) $^{b}$

Tonal

6

Ringing

2

Hissing

3

Tinnitus Ear

Bilateral

11

Left

Right

a Participants matched the $5 \mathrm{kHz} 40-\mathrm{Hz}$ AM training stimulus to the loudness of a $2 \mathrm{kHz} 40-\mathrm{Hz}$ AM $65 \mathrm{~dB}$ SPL sound used in earlier research with young normal hearing subjects Gander et al. (2010b).

${ }^{b}$ Before auditory training.

sound files delivered by the Tinnitus Tester (Roberts et al., 2008). At the outset of training participants rated their tinnitus loudness at 57.1 ( $\mathrm{SE}=6.21$ ) on the Borg CR100 scale of the Tinnitus Tester, which is near the midpoint between of "Loud" and "Very Loud" on this scale. The mean tinnitus spectrum and loudness matches of the participants measured by the Tinnitus Tester are reported in Figures $\mathbf{2 B}$ and 2C, respectively. The training frequency of $5 \mathrm{kHz}$ was 1.5 octaves above the audiometric edge of $2 \mathrm{kHz}$ and in the range of the tinnitus frequency spectrum for this group.

\section{BEHAVIORAL PERFORMANCE}

Psychophysical functions determined for sessions 1,4 , and 7 from the collapsed data of the tinnitus and control groups are presented for each group in Figure 3A. Performance improved rapidly from the first to the fourth session, with smaller improvements occurring in the later sessions. THs determined from psychophysical functions calculated for each participant and session confirmed this trend (Figure 3B) giving a main effect of

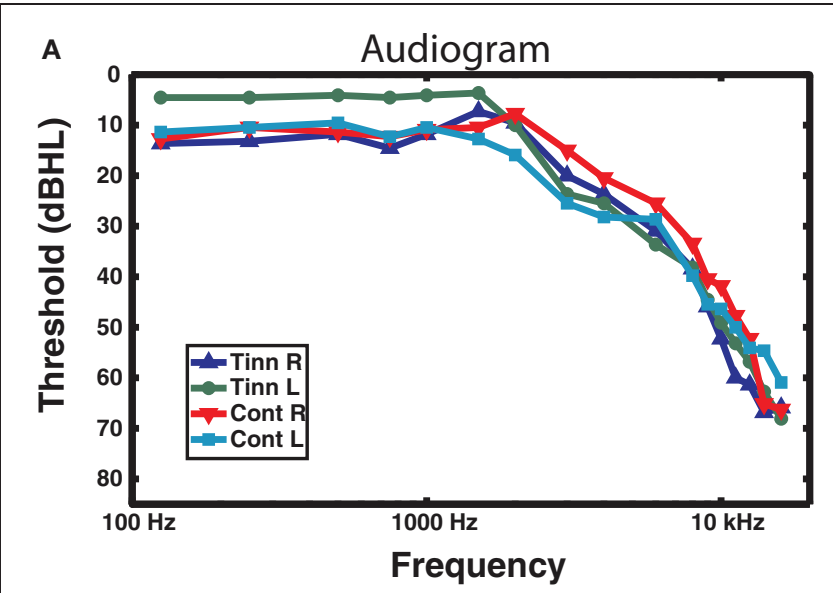

B

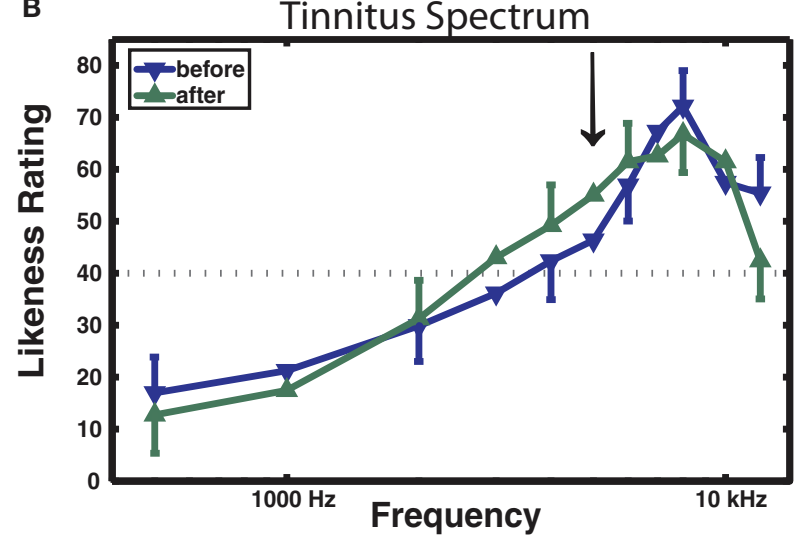

C

Loudness Match

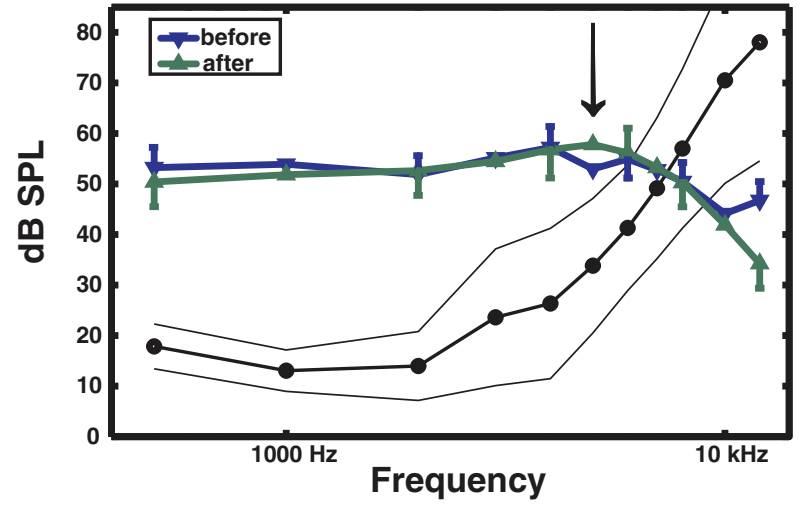

FIGURE 2 | Audiometric and psychoacoustic measures. (A) Audiogram for each group, right and left ears shown separately. For clarity, error bars are omitted (group main effect $F<1$ ). (B) Tinnitus spectrum (likeness ratings) before and after training in the tinnitus group, from the Tinnitus Tester software (Roberts et al., 2008). A likeness rating of 40 (broken line) corresponds to sounds beginning to resemble the individual's tinnitus (the lower boundary of the tinnitus spectrum; Roberts et al. (2008). The arrow denotes the $5 \mathrm{kHz}$ training frequency. (C) Loudness matches from the Tinnitus Tester, before and after training. The line connected by dots denotes the group averaged audiogram converted from $\mathrm{dB} H \mathrm{HL}$ to $\mathrm{dB} S \mathrm{SP}$ (thin lines denote between-subject 95\% confidence limits). The difference between the audiogram and the loudness matches gives an approximate estimate of the loudness of the tinnitus in dB SL (Roberts et al., 2008). Error bars shown in panels (B) and (C) are the mean within-subject standard error (1 SE). 


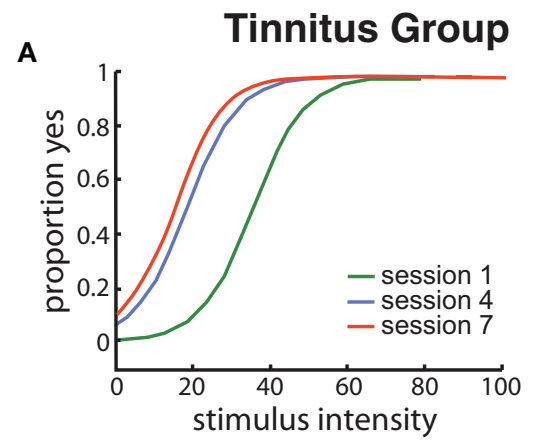

Control Group

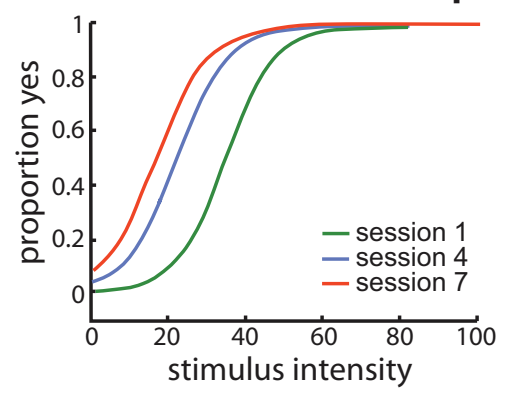

B

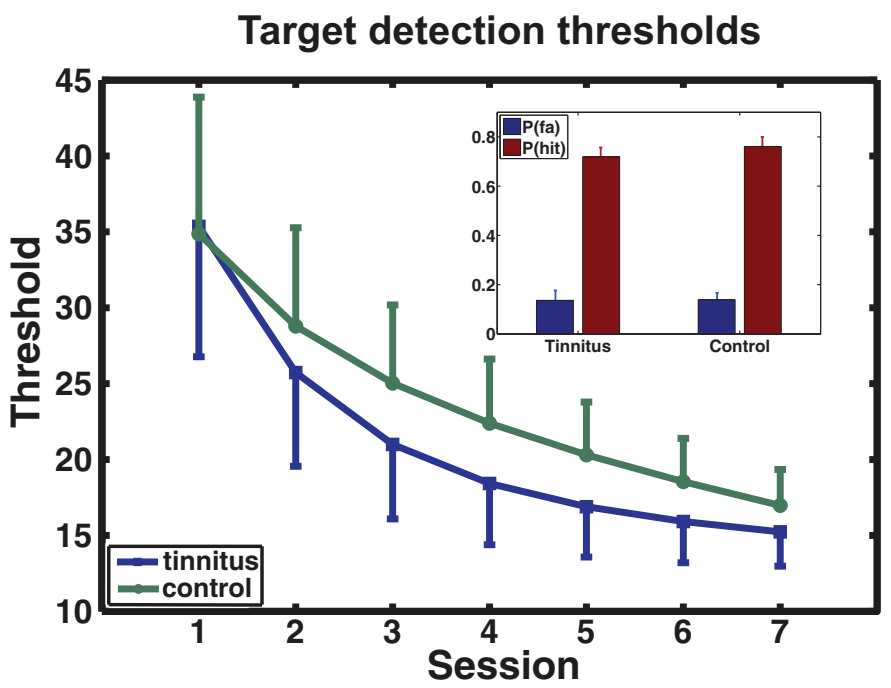

FIGURE 3 | Behavioral performance. (A) Group averaged psychophysical functions are shown for sessions 1, 4, and 7, separately for each group. (B) Changes in target detection THs over sessions for each group. The model used for curve fitting of each participant's data applied a negative exponential (direction determined by the data; see Materials and Methods). Error bars give the mean within-subject standard error (1 SE). The inset shows the mean hit and false alarm rates for each group. sessions $\left[F_{(6,114)}=11.7, p=0.00\right]$ while factors involving group were not significant ( $p$ 's $>0.68)$. $P$ (Hit) exceeded $P(\mathrm{FA})$ for every participant $\left[F_{(1,20)}=289, p<0.0001\right]$ with no group difference on either measure (see inset, Figure 3B).

\section{ASSR PHASE}

ASSR phase for all participants on the first passive block fell within an arc of $123^{\circ}$ centered at $161.9^{\circ}$ with respect to the zerocrossing in the stimulus waveform. Individual differences in ASSR phase were reliable giving a test-re-test correlation between passive blocks on days 1 and 7 of $r=0.91$ in the tinnitus group and $r=0.83$ in controls ( $r=0.86$ overall, $p<0.01$ ). Mean phase in the tinnitus group on the first passive block $\left(168.6^{\circ}, \mathrm{SD}=\right.$ 34.2) did not differ significantly from that of controls $\left(155.2^{\circ}\right.$, $\mathrm{SD}=19.3$ ).

Changes in ASSR phase across sessions are depicted separately for the tinnitus and control groups in Figure 4A. Following Gander et al. (2010b) phase was normalized within each group by dividing the data of each subject by their respective group mean for the passive blocks of day 1, giving a common reference point (1.0) from which to evaluate effects of training and active/passive blocks. In the control group ASSR phase decreased progressively over training sessions $\left[F_{(2,20)}=6.54, p=0.006\right]$, in agreement with results obtained previously for young normal hearing subjects trained by Gander et al. (2010b) with a $2 \mathrm{kHz}$ $40-\mathrm{Hz}$ AM sound. The phase change in the control group (reflecting a shortened time delay between zero-crossings in the stimulus and response waveforms) was more pronounced on active blocks, but neither the main effect of block $(p=0.35)$ nor its interaction with sessions $(p=0.60)$ reached significance. The phase shift averaged $-10.1^{\circ}$ on active blocks, which is similar to mean shifts of $-14.7^{\circ}$ and $-12.9^{\circ}$ on these blocks observed in Groups E and $\mathrm{C}$, respectively, of Experiment 2 by Gander et al. (2010b). On the other hand, neither the main effect of sessions $\left[F_{(2,20)}=1.63\right.$, $p=0.22]$ or blocks $\left[F_{(1,10)}=1.31, p=0.28\right]$ nor their interaction $\left[F_{(2,20)}=0.79, p=0.46\right]$ were significant in the tinnitus group. When the tinnitus and control groups were entered into the same analysis a main effect of sessions was found $\left[F_{(2,40)}=\right.$ $5.08, p=0.011]$, but the interaction of group with sessions did not reach significance $(p=0.20)$. However, the phase shift seen in tinnitus participants on active blocks (mean difference day 7 - day $1=-2.1^{\circ}$ ) was significantly smaller when contrasted to that of the three above mentioned non-tinnitus groups (mean shifts of $-10.1,-14.7$, and $-12.9^{\circ}$, respectively, $t_{(38)}=2.43$, $p=0.019)$. These results suggest that the presence of tinnitus may have interfered with normal remodeling of ASSR phase by the training procedure.

Two further analyses were undertaken to assess performance in the tinnitus group. In the first analysis, the before/after change in ASSR phase (day 7 - day 1) was determined for each tinnitus and control subject on active blocks and are rank ordered by magnitude in Figure 4B. Several tinnitus subjects expressed phase shifts overlapping with those of control subjects; however, phase shifts in the control group were less variable and for each 


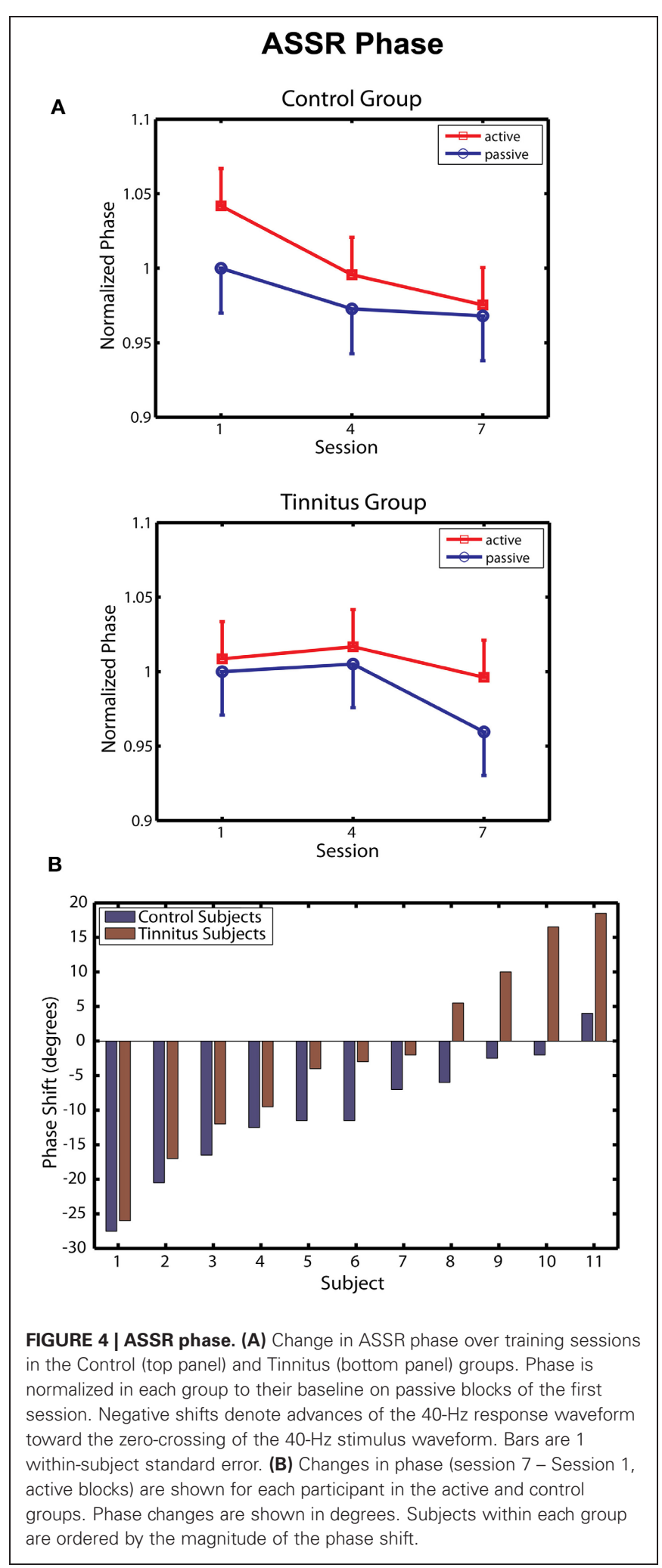

pairwise comparison were in the direction of a phase decrease for controls relative to individuals with tinnitus. Overall ASSR phase decreased in $91 \%(10 / 11)$ of the control subjects of this study and in $90 \%(18 / 20)$ of normal hearing subjects reported by Gander et al. (2010b), compared to 63\% (7/11) of tinnitus subjects reported here. Figure 4B further shows that the group difference was affected by an increasing delay between the response and stimulus waveforms (phase increases) that were seen in some participants with tinnitus. It may be noteworthy that the peak of the tinnitus spectrum in these participants (mean $=8.0 \mathrm{kHz}$ ) tended to be closer to the $5 \mathrm{kHz}$ training stimulus than was the case for subjects showing phase decreases (mean $=8.71 \mathrm{kHz}$; $r=-0.59, p<0.06$ ). Except for this tendency, no correlate was found (hearing THs, stimulus levels, other tinnitus attribute, or brain response) that might explain the performance of these individual tinnitus subjects. In a second analysis, we attempted to contrast within-session phase shifts between the first two and last two active blocks of each session, between our tinnitus and control groups. On average phase decreased $-7.50^{\circ}$ within sessions in the control group compared to a phase increase of $2.65^{\circ}$ in tinnitus subjects, but this difference did not reach significance ( $p=$ 0.34 ). Thus, within the limits of this analysis (signal-to-noise ratio is greatly reduced in sub-block analyses) it did not appear that the tinnitus group displayed phase decreases within sessions that were reset by neural activity related to tinnitus between sessions.

\section{ASSR AMPLITUDE}

ASSR amplitude measured as total field power (128 electrodes) varied widely across subjects from $467 \mu \mathrm{V}^{2}$ to $22,132 \mu \mathrm{V}^{2}$, averaging $1871 \mu \mathrm{V}^{2}(\mathrm{SD}=1074)$ in the tinnitus group and $4192 \mu \mathrm{V}^{2}$ $(\mathrm{SD}=6081)$ in controls on the passive blocks of the first session. Although mean field power was thus 2.2 times larger in the control group than in the tinnitus group on passive blocks, this difference did not reach significance $[t(20)=1.25, p=0.22]$. However, individual differences in ASSR amplitude were highly reliable giving test-re-test correlations between sessions 1 and 7 of $r=0.99$ and 1.0 in the tinnitus and control groups, respectively, in agreement with unpublished observations by Gander et al. (2010b). These differences likely reflect individual variation in the anatomy of Heschl's gyrus, the orientation of ASSR generators in this region, and the summed neural activity of ASSR sources across reversing tonotopic maps sharing a low frequency border situated laterally in A1 (Kaas and Hackett, 2000; Formisano et al., 2003).

Changes in ASSR amplitude across sessions are depicted separately for the tinnitus and control groups in Figure 5A. Following Gander et al. (2010b) ASSR amplitude was normalized by dividing the data of each subject by their respective group mean for the passive blocks of day 1. In agreement with previous findings (Gander et al., 2010b) ASSR amplitude was larger on active blocks where subjects performed the task than on passive blocks where they did not, in the control group $\left[F_{(1,10)}=4.85, p=0.05\right]$ as well as in the tinnitus condition $\left[F_{(1,10)}=5.09, p=0.03\right]$. In controls no further effects were found, which is consistent with Gander et al. (2010b) where ASSR amplitude did not increase over four sessions of training with EEG measurement although an increase was detected in a concluding tenth session where EEG was again measured. The picture in the tinnitus group was different. Here ASSR amplitude increased over sessions, first on 


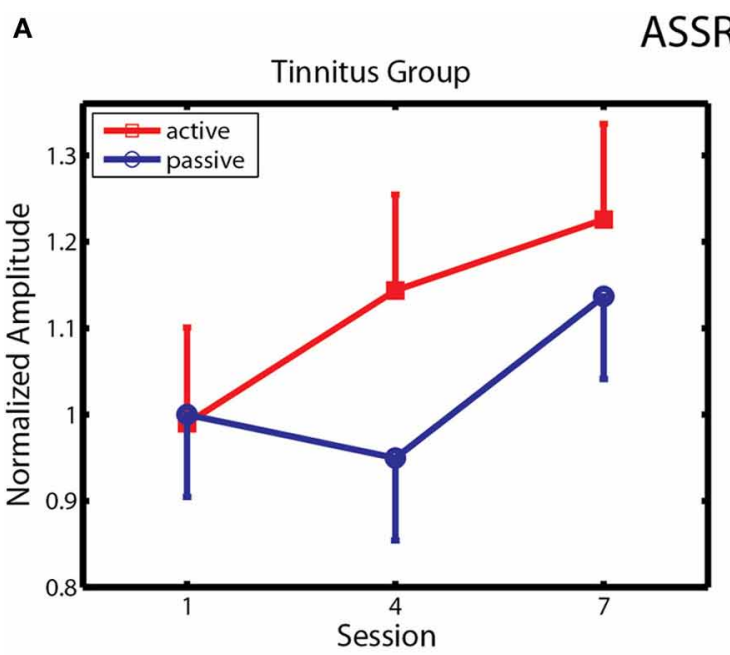

\section{ASSR Amplitude}

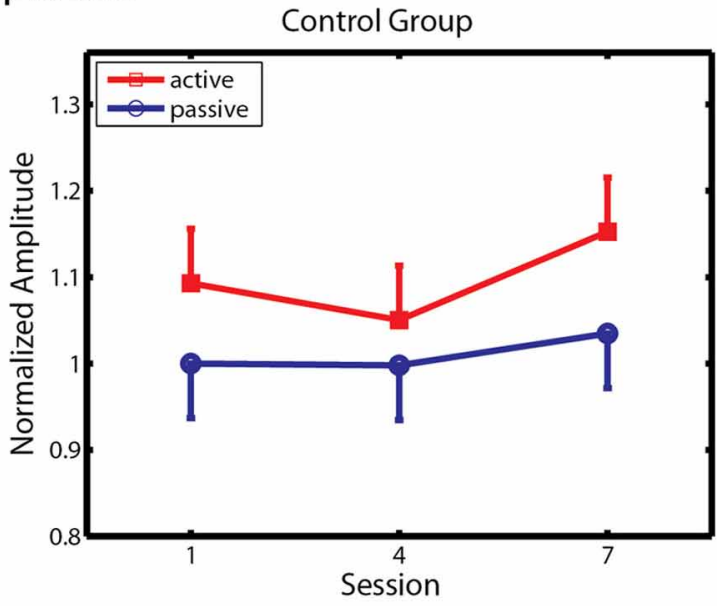

B

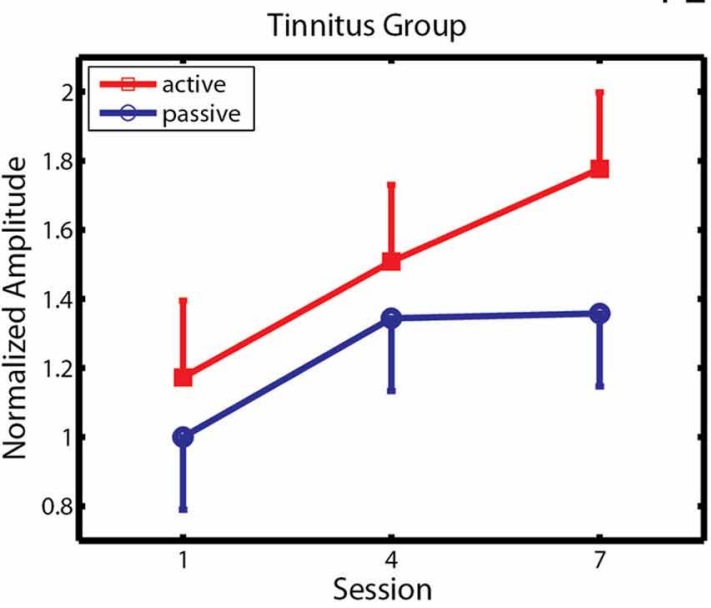

FIGURE 5 | ASSR amplitude and P2 amplitude. (A) Changes in ASSR amplitude over training in the Tinnitus (left panel) and Control (right panel) groups, on active and passive blocks. (B) Changes in P2 amplitude are
P2 Amplitude

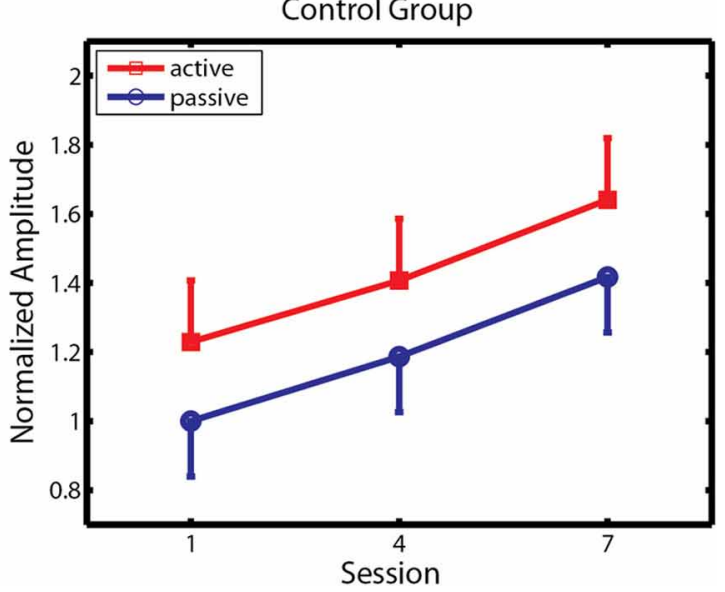

similarly depicted. Response amplitude is normalized within each group to amplitude on passive blocks of the first session. Bars denote 1 within-subject standard error. active and then on active and passive blocks, giving an interaction of sessions with block $\left[F_{(2,20)}=4.68, p=0.022\right]$ as well as the main effect of block mentioned above. Post-hoc contrasts found that ASSR amplitude was larger on the active and passive blocks of session 3 compared to the corresponding blocks of session 1, and on the active blocks of session 2 compared to the active and passive blocks of session 1 (all $p$ 's $<0.006$, LSD tests). When the tinnitus and control groups were entered in the same analysis a main effect of block was found $\left[F_{(1,20)}=10.84, p=0.003\right]$ as well as a three-way interaction of session, block, and group $\left[F_{(2,40)}=4.56, p=0.016\right]$ which reflected the growth of ASSR amplitude preferentially on active trials in the tinnitus group. Thus, ASSR amplitude was larger on active than passive blocks in both groups but increased over seven sessions of auditory training only in the tinnitus condition.

\section{P2 AMPLITUDE}

P2 amplitude ranged from $-1.87 \mu \mathrm{V}$ to 2.57 across all participants, averaging $0.48 \mu \mathrm{V}(\mathrm{SD}=0.89)$ in the tinnitus group and $0.57 \mu \mathrm{V}(\mathrm{SD}=1.17)$ in controls (group difference not significant). Individual differences were reliable across active blocks on days 1 and 7 within the control group $(r=0.84, p<0.05)$ but less so in the tinnitus group ( $r=0.31$, not significant) although the preceding $\mathrm{N} 1$ response was reliable in both groups $(r=0.65$ and 0.70 in controls and tinnitus, respectively, both $r$ 's $p<0.05)$.

Changes in P2 amplitude across sessions are depicted separately for the tinnitus and control groups in Figure 5B. Again, amplitude was normalized by dividing the data of each subject by their respective group mean for the passive blocks of Day 1. P2 amplitude increased progressively over training sessions in both groups on active and passive blocks, with larger P2 amplitude 
seen on active trials in each group. Main effects of session were found in both the control $\left[F_{(2,20)}=7.61, p=0.003\right]$ and tinnitus $\left[F_{(2,20)}=7.67, p=0.003\right]$ groups, confirming a training effect on P2 amplitude. The main effect of block was also significant in the control group $\left[F_{(2,20)}=5.35, p=0.04\right]$ and nearly so in the tinnitus group $\left[F_{(1,20)}=3.23, p=0.10\right]$. Analysis of the combined groups revealed a main effect of sessions $\left[F_{(2,40)}=\right.$ $14.8, p=0.00001]$ and of block $\left[F_{(1,20)}=7.80, p=0.011\right]$ and no other effects. Thus, P2 amplitude remodeled normally in tinnitus subjects, equaling the performance of their age and hearing matched controls. The changes observed in these groups are comparable to those reported in studies by Gander et al. (2010b) and others (Tremblay et al., 2001; Reinke et al., 2003; Bosnyak et al., 2004; Sheehan et al., 2005; Alain et al., 2007; Ross and Tremblay, 2009) showing increased P2 amplitude after auditory training in normal hearing individuals. No main effects or interactions involving group, sessions, or block were found for P2 latency, which averaged $203.9 \mathrm{~ms}$ overall $(\mathrm{SD}=15.2 \mathrm{~ms})$ on active blocks of the first session.

\section{OTHER TRANSIENT RESPONSES}

The transient responses P1 (mean latency $47.4 \mathrm{~ms}$ ), N1 (mean latency $109.8 \mathrm{~ms}$ ), N2 (mean latency $326.0 \mathrm{~ms}$ ), and the auditory sustained response (SR) (350-900 ms) were also analyzed for each subject and group. No effects involving sessions, block or group were found on the latency of P1, N1, and P2 transient responses. Neither did the amplitude of P1, N1, and N2 responses or the SR change significantly over sessions in either group. On the other hand, P1 amplitude and N2 amplitude were significantly larger on active compared to passive blocks in both groups, as was the SR in the tinnitus group; main effects of block were found for each response when the groups were combined ( $p<0.004,0.008$, and 0.001 , respectively, for P1, N2, the SR). N1 amplitude was also larger on active than passive blocks, but only in the control group (block by group interaction $p=0.041)$. When passive blocks alone were considered where participants performed no task, N1 tended to be larger in tinnitus subjects $(-3.02 \mu \mathrm{V})$ than in controls $(-2.26 \mu \mathrm{V} ; p=0.077$, LSD test).

\section{EFFECTS OF TRAINING ON TINNITUS}

Four measurements of tinnitus were taken from tinnitus participants in the present study, namely; (i) ratings of tinnitus awareness and loudness recorded three times within each training session; (ii) psychoacoustic measurements of tinnitus loudness (by sound level matching) and the tinnitus spectrum taken by the Tinnitus Tester of Roberts et al. (2008) before and after training; (iii) ratings of tinnitus loudness on a Borg CR100 scale by the Tinnitus Tester; and (iv) measurement of the participant's reaction to tinnitus by the THQ before and after training.

The first of these measures is reported in Figure 6. Tinnitus awareness (panel A) diminished across the three measurements taken within each session (main effect of measurement $\left[F_{(2,20)}=\right.$ 5.45, $p<0.037]$. The interaction of measurement with sessions was also significant $\left[F_{(12,120)}=4.28, p<0.00001\right]$, reflecting the larger within-session change seen on the first day of training.

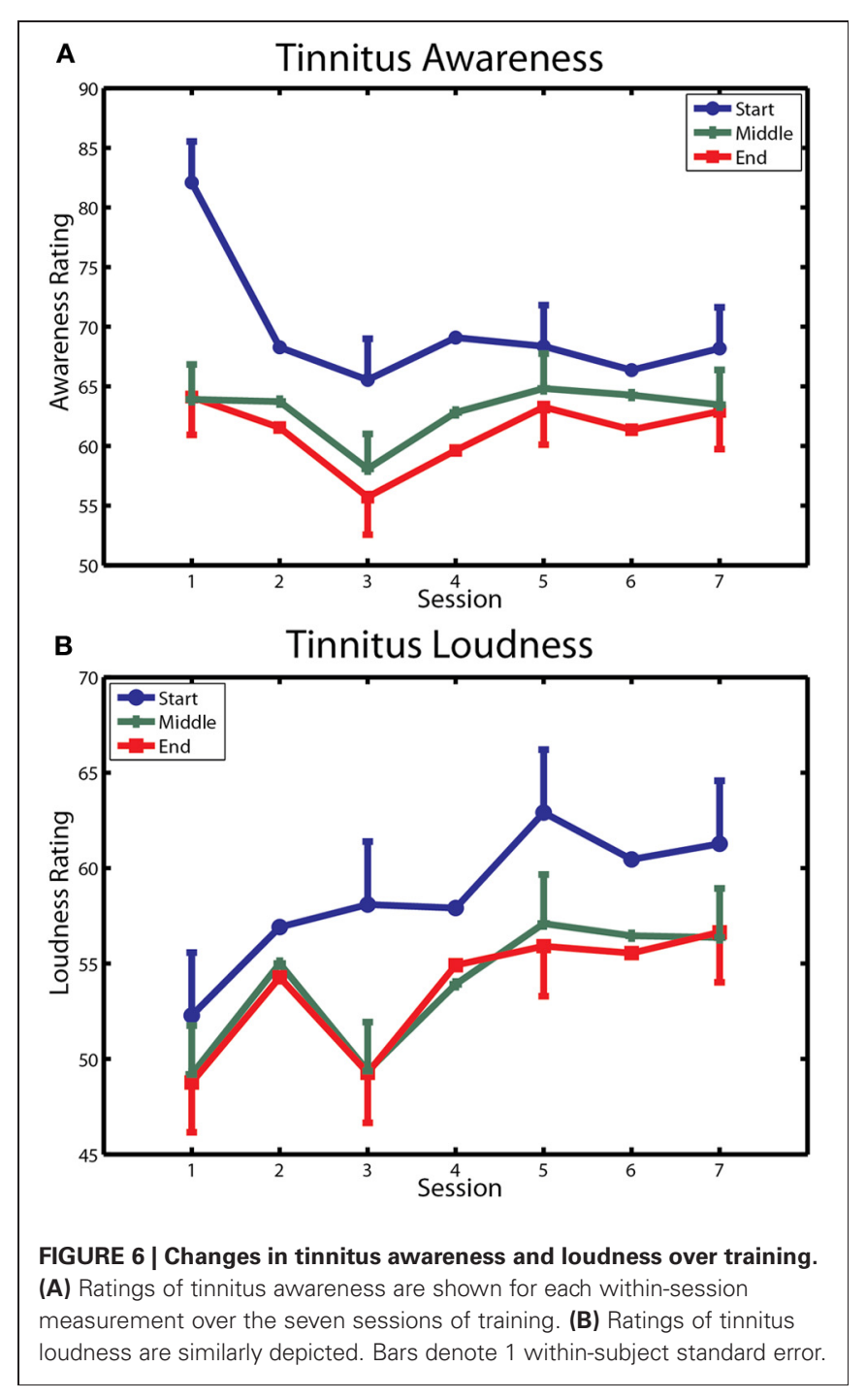

Tinnitus awareness averaged over measurements of the last session was 64.8 ( $\mathrm{SD}=19.4)$, which was above the midpoint between "Aware" and "Very Aware" on the rating scale used for these measurements. Tinnitus loudness (bottom panel) decreased non-significantly within sessions $(p=0.17)$ but over days showed an upward trend that approached significance (main effect of sessions $p=0.09$ ). The overall mean loudness rating of 58.1 ( $\mathrm{SD}=$ 20.4) on the last day of training corresponded to a rating between "Loud" and "Very Loud" on the Borg CR 100 scale used for these ratings.

Figure 2B shows the tinnitus spectrum (likeness ratings) and Figure 2C tinnitus loudness matches taken before and after training by the Tinnitus Tester. For the likeness ratings a significant a main effect was found for frequency $\left[F_{(10,100)}=14.7, p=0.000\right]$ confirming earlier results that sound frequencies in the region of hearing loss are typically judged to resemble tinnitus percepts (Noreña et al., 2002; Roberts et al., 2008; Sereda et al., 2011; Zhou et al., 2011). The main effect of before/after was not significant, but the interaction of frequency with before/after came 
close $\left[F_{(10,100)}=1.77, p=0.074\right]$ and was significant when the frequencies of $5 \mathrm{kHz}$ (the training frequency) and $8 \mathrm{kHz}$ were used for the analysis $\left[F_{(1,10)}=10.7, p=0.008\right]$. The same analyses applied to the loudness matches revealed no significant effects. Thus, while auditory training had no effect on loudness matches, the tinnitus spectrum was affected, with $5 \mathrm{kHz}$ rated more highly and $8 \mathrm{kHz}$ less highly as a component of tinnitus after training than before training. Figure $2 \mathrm{C}$ also includes the mean audiogram of the tinnitus participants converted from $\mathrm{dB}$ HL to dB SPL. Tinnitus loudness matches referenced to the audiogram before training (which gives an approximation of initial tinnitus loudness in $\mathrm{dB} S \mathrm{SL}$ ) revealed a significant effect of frequency $\left[F_{(10,100)}=21.3, p=0.000\right]$, confirming previous reports (Henry and Meikle, 2000; Roberts et al., 2008) that tinnitus loudness matches are greater when the sound frequencies used for matching are below rather than in the spectrum of the tinnitus (in the present data, $40.8 \mathrm{~dB}$ SL at $1 \mathrm{kHz}$ compared to $3.9 \mathrm{~dB}$ SL at $7 \mathrm{kHz}$ ).

The effect of auditory training on the THQ, and on loudness ratings on the Borg CR100 scale included in the Tinnitus Tester, are summarized in Table 2, where measurements taken before and after auditory training are contrasted. For completeness, this table also reports before/after loudness matches to the $1 \mathrm{kHz}$ sound taken from the Tinnitus Tester (in dB SPL, these data from Figure 2C) and ratings of tinnitus loudness and awareness taken within sessions 1 and 7 of auditory training (these data from Figures 6B and 6A, respectively). Correlations between the first (Before) and second (After) measurements for each variable are reported in the last column of Table 2 and give a conservative estimate of test-re-test reliability for each measure, keeping in mind that effects of auditory training (if present) could attenuate them. The correlations ranged from 0.49 to 0.98 and in most instances were statistically significant indicating the presence of reliable individual variability. Nonetheless only Factor 2 of the THQ showed a significant change, in this case indicating that difficulties of hearing in noise were reported to be slightly worse after auditory training than before. Other measures (total score on the THQ, Factors 1 and 3 of the THQ, the tinnitus rating and loudness match from the Tinnitus Tester, and tinnitus awareness assessed by the Borg CR100 scale within sessions) were in the direction of a beneficial effect of auditory training on tinnitus, but none of these differences reached significance.

\section{CORRELATIONS AMONG EEG RESPONSES AND THEIR RELATION TO HEARING FUNCTION AND TINNITUS}

Two between-subject correlational analyses were conducted. The first analysis examined relationships among training-induced changes in ASSR amplitude, ASSR phase, and P2 amplitude, and the relationship of these changes to the effects of active and passive blocks on the responses. When the tinnitus and control groups were combined, changes in ASSR amplitude produced by training and by active versus passive blocks were correlated with baseline ASSR amplitude on the passive blocks $(r=0.65$ and 0.79 , respectively, $p<0.05$ ), reflecting larger contributions to these effects by participants with larger ASSR baseline amplitudes. This pattern was evident within each group separately. In the tinnitus group changes in P2 amplitude with training correlated with changes in ASSR amplitude $(r=0.781, p<0.05)$, but changes in P2 amplitude with training did not correlate with ASSR amplitude changes in the control group or with any other brain measure in either group.

The second analysis examined the relationship of changes in EEG responses to (1) the loudness of the training stimuli and to hearing THs at $5 \mathrm{kHz}$ in the tinnitus and control groups separately and in the combined sample, and (2) to the tinnitus measures of Table 2 in the tinnitus group. Table 1 shows that the tinnitus and control groups were well matched for hearing THs at $2 \mathrm{kHz}$ and $5 \mathrm{kHz}$ and for stimulus level (no group differences were found on these measures). ASSR amplitude, ASSR phase, and P2 amplitude measured during the passive baseline did not correlate with sound THs at $2 \mathrm{kHz}$ or $5 \mathrm{kHz}$ or with stimulus level, nor did changes in these brain responses over training correlate significantly with sound THs or stimulus level within each group separately or in the combined sample. Sound THs at $2 \mathrm{kHz}$ and $5 \mathrm{kHz}$ increased with age $(r=0.83$ and 0.92 , respectively, both $p<0.05)$, but age did not correlate with effects of training or of active/passive blocks on any brain response and did not differ significantly between the tinnitus and control groups (see Table 1). Correlations between changes in ASSR amplitude, ASSR phase, and P2 amplitude over training and changes in the tinnitus measurements of Table 2 were not significant.

Table 2 | Tinnitus Handicap Questionnaire and Tinnitus Loudness.

\begin{tabular}{|c|c|c|c|c|}
\hline & Before & After & Difference & Correlation \\
\hline \multicolumn{5}{|l|}{ THQ SCORES } \\
\hline THQ Total score & $48.9(6.66)$ & $46.4(7.48)$ & $-2.4(3.12)$ & $0.90^{*}$ \\
\hline Factor 1 (Physical, emotional, social consequences of tinnitus) & $51.1(7.63)$ & $46.1(8.84)$ & $-4.9(4.26)$ & $0.89^{*}$ \\
\hline Factor 2 (Tinnitus effects on hearing abilities) & $40.8(8.71)$ & $45.8(8.52)$ & $5.1(2.06)^{* *}$ & $0.98^{*}$ \\
\hline Factor 3 (Patient's views on tinnitus) & $56.8(5.00)$ & $48.7(4.38)$ & $-8.1(4.57)$ & $0.59^{\dagger}$ \\
\hline \multicolumn{5}{|l|}{ TINNITUS LOUDNESS AND AWARENESS } \\
\hline Loudness rating from Tinnitus Tester (Borg CR100 scale) & $57.1(6.21)$ & $52.6(7.13)$ & $-4.6(3.09)$ & $0.91 *$ \\
\hline Loudness match to $1 \mathrm{kHz}$ sound from Tinnitus Tester (dB SPL) & $53.9(6.32)$ & $51.8(4.51)$ & $-2.1(3.46)$ & $0.85^{*}$ \\
\hline Loudness rating Session 1 and Session 7 (Borg CR100 scale) & $50.1(6.10)$ & $58.1(6.28)$ & $8.0(4.36)$ & $0.75^{*}$ \\
\hline Awareness rating Session 1 and Session 7 (Borg CR100 scale) & $70.0(5.18)$ & $64.9(5.86)$ & $-5.2(5.60)$ & 0.49 \\
\hline
\end{tabular}

${ }^{*} p<0.01$ *** $^{*} p<0.05 ;^{\dagger} p<0.10$. 


\section{DISCUSSION}

Sensory training therapies for tinnitus are based on the assumption that, notwithstanding neural changes related to tinnitus, auditory training can alter the response properties of neurons in auditory pathways, as has been demonstrated in normal hearing individuals (Gander et al., 2010b). To address this question, we investigated whether brain changes induced by sensory training in tinnitus sufferers and measured by EEG are similar to those induced in age and hearing level matched individuals without tinnitus trained on the same auditory task. Auditory training was given using a sound that was in the tinnitus frequency region of the tinnitus subjects. We found that P2 amplitude increased with training equally in participants with tinnitus and in control subjects, suggesting normal remodeling non-primary (A2) auditory regions in tinnitus. However, training-induced changes in the $40 \mathrm{~Hz}$ ASSR, which localizes to sources in posteriormedial Heschl's gyrus (A1), differed between the tinnitus and control groups. Training-induced changes ASSR phase which reflect changes in temporal population activity expressed in A1 were normal in control participants; the time delay between the response and stimulus waveforms decreased by about $-10^{\circ}$ in this group. However, ASSR phase did not change significantly with training in the tinnitus group. On the other hand, ASSR amplitude increased with training in the tinnitus group, whereas in controls no changes were seen in this response attribute over training. Effects of auditory training on tinnitus loudness were not significant, although an effect on the tinnitus spectrum was detected.

These results suggest that neural changes related to tinnitus altered how neural plasticity was expressed in A1 but not A2 auditory cortex. We discuss auditory plasticity in normal hearing individuals and then consider how the presence of tinnitus may have affected the expression of neural plasticity in auditory pathways. In a concluding section limitations on our findings are discussed.

\section{AUDITORY REMODELING IN NORMAL HEARING}

The first reported study of auditory training using the $40 \mathrm{~Hz}$ ASSR (Bosnyak et al., 2004) required that normal hearing subjects discriminate between a standard stimulus consisting of a $40 \mathrm{~Hz}$ AM $2 \mathrm{kHz}$ carrier frequency and comparison stimuli using carriers that were either $200 \mathrm{~Hz}$ higher or lower than the standard. The phase delay between the ASSR response and stimulus waveforms shortened in a brief interval (150-400 ms) following stimulus onset over 15 sessions of training, and was greater for the trained standard stimulus $(2 \mathrm{kHz})$ than for untrained comparison stimuli. However, no change was detected in ASSR amplitude even though behavioral performance improved over the lengthy training experience. Because competitive interactions among the different carrier frequencies during frequency discrimination may have prevented expansion of the tonotopic representation for the standard sound (Kilgard et al., 2001), the stimulus procedure of the current study was designed to deliver extensive experience of only a single carrier frequency over training, and to deliver target events randomly in the second half of the stimulus so that sustained attention was required. Using this procedure Gander et al. (2010b) found that the magnitude of the phase change increased over ten sessions of training, expressed throughout the duration of the training stimulus, and correlated with behavioral performance over the training sessions. Effects on ASSR amplitude were, however, more variable. An increase in this response was not observed until the tenth session of training, and the change in ASSR amplitude over sessions did not correlate with behavioral performance while the phase shift did. No increase in ASSR amplitude was seen in a control group that received two sessions of training separated by six weeks, although the ASSR phase shift occurred in this group dissociating the two measures in agreement with previous findings.

A further difference between ASSR phase and ASSR amplitude in the research of Gander et al. (2010a,b) concerned the effects of task attention (active and passive sound exposure) on the two response attributes. ASSR amplitude was larger on active blocks than on passive blocks in the Gander et al. (2010b) studies, as was true in the tinnitus and control groups of the present study. Parallel research disambiguating the effects of auditory attention from button pressing and correctness feedback on active blocks showed that attention to the trained sounds was the source of the ASSR amplitude increase (Gander et al., 2010a). However, attention was found to have no effect on ASSR phase in these studies. Gander et al. (2010b, Experiment 1) also found that changes in phase with auditory training appeared in equal magnitude in a group that performed the task for two sessions under conditions of attention as in a group that heard the same $40-\mathrm{Hz}$ AM sounds presented passively while they watched a silent video with no knowledge of the auditory task. Thus, while attention increases ASSR amplitude (Ross et al., 2004; Gander et al., 2010a), it has no effect on ASSR phase and does not appear to be required for changes in this response attribute with auditory experience.

In order to explain these findings, Gander et al. (2010b) suggested that the ASSR phase shift may reflect stimulus-driven changes in temporal activity that occur in subcortical auditory structures during auditory experience and are inherited by the cortical sources of the ASSR in A1 with little involvement of attention. The temporal response properties of subcortical auditory neurons are known to be modified by experience with complex speech and musical sounds (Song et al., 2008; Tzounopoulos and Kraus, 2009) indicating that neural plasticity is expressed in these structures. In subsequent research with the same stimulus procedure, Baynton (2010, unpublished results) obtained phase shifts that were as evident in children aged 5-8 years as in older age cohorts aged 18-25 years, consistent with an early form of stimulus-driven plasticity operating in auditory pathways. ASSR amplitude did not increase with training in any age group in this study, but this variable increased during auditory attention (active compared to passive blocks) in cohorts aged 13-15 years and older although not at younger ages. It has been suggested (Gander et al., 2010b) that increases in ASSR amplitude on active blocks may reflect activation of the basal forebrain cholinergic system that performs some of the functions of attention (Sarter et al., 2005) by distributing acetylcholine to the neocortical mantle thus making cortical neurons more sensitive to their afferent inputs (Metherate and Ashe, 1993). Animal data indicate that this system does not mature until puberty (Kiss and Patel, 1992), 
which aligns with Baynton's results where modulation of ASSR amplitude by active and passive blocks did not reach significance until age $13-15$ years.

\section{AUDITORY REMODELING IN TINNITUS}

To allow comparison with earlier results obtained from normal hearing subjects trained with a $2 \mathrm{kHz} 40-\mathrm{Hz}$ AM sound, the current study used the training method of Gander et al. (2010b) substituting a $5 \mathrm{kHz} 40-\mathrm{Hz}$ AM sound which was in the tinnitus frequency region of the tinnitus subjects. Individuals with tinnitus and control subjects of comparable age and $\mathrm{TH}$ hearing function were studied. The results from the control group with regard to ASSR phase and amplitude were in agreement with earlier findings (Gander et al., 2010b) and are consistent with the interpretation suggested for those findings. However, the results from participants with tinnitus were different, and invite interpretation from current knowledge regarding neural changes associated with hearing loss and tinnitus.

One interpretation of impaired phase plasticity in the tinnitus group is that remodeling of phase occurred normally within sessions, but that persisting neural changes associated with tinnitus (these not present in controls) reset the phase changes between sessions in the tinnitus group. However, within the limits of our data, analyses of within-session phase shifts in the tinnitus and control groups did not support this interpretation. Alternatively, neural population activity associated with tinnitus may have interfered with the processing that occurs in A1 or in subcortical structures projecting to this region, in the tinnitus group. This possibility received limited but provocative support from the observation that phase shifts opposite to the normal phase change occurred in participants whose dominant tinnitus frequency was comparatively closer to the trained $5 \mathrm{kHz}$ sound $(r=-0.59, p<0.06)$. This relationship could reflect an interaction between tinnitus-related synchronous neural activity occurring in the dominant tinnitus frequency region (Weisz et al., 2007) and synchronous neural activity driven by the $5 \mathrm{kHz}$ $40-\mathrm{Hz}$ AM training stimulus. A third possibility is that because ASSR amplitude increased over training in the tinnitus subjects suggesting an increased cortical representation in A1 for the trained sound, it may be considered that this effect obstructed changes in ASSR phase in this group. The relationship of changes in ASSR amplitude to changes in ASSR phase between tinnitus subjects was in the direction of such an effect $(r=-0.328)$ but did not reach significance. It is of interest that ASSR amplitude increased significantly with training in the tinnitus group, but not in the control group of this study. Enhanced remodeling of ASSR amplitude could be consequent on inhibitory deficits that occur in central auditory structures with hearing loss (Scholl and Wehr, 2008) and tinnitus (Wang et al., 2009), which could permit additional neurons to be recruited into a representation for the trained sound. If this hypothesis is provisionally accepted, it implies that similar inhibitory changes were absent in controls who did not have tinnitus although they had a similar level of hearing loss. The absence of tinnitus in controls could signal comparatively better preserved inner hair cell function in these individuals despite outer hair cell damage contributing to their TH shifts (Kujawa and Liberman, 2009).
In contrast to group differences in remodeling of ASSR phase and amplitude, which reflect group differences in neural processing expressed in the region of A1, P2 amplitude (reflecting neural activity in non-primary regions) increased normally over training in both groups. The amplitude of the preceding N1 response did not change with training, indicating an effect specific to the P2 component of the N1/P2 waveform in agreement with earlier findings (e.g., Bosnyak et al., 2004; Ross and Tremblay, 2009). P2 amplitude was also larger on active compared to passive blocks pointing to its modulation by attention, but this effect did not interact with groups or with the effect of training on P2. The effect of attentional performance on P2 amplitude seen here agrees with observations by Gander et al. (2010b), although modulation of P2 amplitude by attention on other types of task is not always seen (cf. Hillyard et al., 1973; Carpenter et al., 2002) possibly relating to procedural variables and their effect on the preceding polarity-opposite N1. In contrast, enhancement of P2 amplitude by auditory training is a robust finding reported by many studies cited above. Changes in P2 amplitude induced by training in the control group of the present study and in earlier research by Gander et al. (2010b) were uncorrelated between-subjects with changes in ASSR amplitude, suggesting that these two responses reflect independent events occurring in auditory pathways. In contrast, in the tinnitus group of the present experiment these effects were correlated between-subjects $(r=0.78, p<0.05)$. These findings suggest that neural activity in A1 (reflected by increased ASSR amplitude in the tinnitus group) may modulate P2 cortical sources in A2, but P2 sources can change independently of events occurring in the auditory core region via parallel thalamocortical projections or top-down pathways.

\section{EFFECTS OF AUDITORY REMODELING ON TINNITUS}

The stimulus procedure of this study was adopted from previous research so that we could compare neural plasticity in individuals with tinnitus and their aged-matched controls with results obtained from normal hearing subjects trained with the same method. There were, however, reasons to suggest that training with sounds in the tinnitus frequency region might also alter tinnitus. In earlier research (Roberts and Bosnyak, 2010a) we found that ASSR amplitude evoked by a $5 \mathrm{kHz} 40-\mathrm{Hz}$ AM sound in the tinnitus frequency region was reduced in individuals with tinnitus compared to hearing level matched controls, while masking in this region (which yielded residual inhibition) restored ASSR amplitude to control levels suggesting modified neural activity in or projecting to A1. Because ASSR amplitude increased during residual inhibition, we were interested to learn whether auditory training with a $5 \mathrm{kHz} 40-\mathrm{Hz}$ AM sound could increase ASSR amplitude in our tinnitus subjects, and if so, diminish their tinnitus percept in the $5 \mathrm{kHz}$ region. For this reason we conducted a thorough assessment of tinnitus before, during, and after training. Consistent with results reported by Roberts and Bosnyak (2010b), non-normalized ASSR amplitude evoked by the $5 \mathrm{kHz}$ $40-\mathrm{Hz}$ AM sound tended to be lower in the tinnitus group compared to controls under passive conditions. However, even though training increased ASSR amplitude in the tinnitus group, the tinnitus percept (likeness rating) did not decrease at $5 \mathrm{kHz}$ but showed an increase relative to $8 \mathrm{kHz}$ instead (Figure 2B). On-line 
ratings of tinnitus loudness also tended to increase over sessions (Figure 6B). However, changes in ASSR amplitude with training did not correlate between-subjects with changes in either of these attributes of tinnitus. Alternatively, changes in the tinnitus spectrum after auditory training could reflect greater familiarity with the dimension of pitch after training. Noreña et al. (2002) observed changes in the shape of the spectrum in a single participant who was trained to discriminate among frequencies around four standard stimuli between 3279 and $6500 \mathrm{~Hz}$. After training the contribution of frequencies above $8 \mathrm{kHz}$ to the tinnitus percept was significantly reduced. Whether this effect reflected a reduction in cortical representation for the untrained sounds or greater familiarity with the trained stimuli could not be decided.

Sound therapies are based on the premise that neural plasticity can be harnessed to ameliorate phantom sound. Our results suggest that the presence of tinnitus may itself affect how neural activity is modified by auditory training in the tinnitus frequency region. Although we did not observe meaningful improvements in tinnitus, it remains possible that sounds with wider bandwidth trained for longer periods could be beneficial. In support of this possibility, treatment studies using the Neuromonics procedure (Davis et al., 2008), which delivers complex low-level sounds covering the tinnitus frequency (hearing loss) region, have reported reductions in tinnitus as well as improved minimum masking and loudness tolerance levels for a subset of tinnitus patients. Long-term exposure to low-level background sound also rescales loudness growth functions in normal hearing individuals (Formby et al., 2003) and, when engineered to cover the hearing loss region, improves loudness tolerance in hyperacusis patients (Noreña and Chery-Croze, 2007). These findings for tinnitus and hyperacusis are congruent with animal research showing that passive exposure to band-pass filtered low-level sound for weeks or longer suppresses neural activity and cortical representations in the exposure frequency band (Pienkowski and Eggermont, 2010).

\section{LIMITATIONS AND QUESTIONS FOR FURTHER STUDY}

Several limitations of the present results for understanding auditory plasticity in normal hearing and in tinnitus remodeling should be noted. One limitation that is the mechanism (or mechanisms) underlying the ASSR phase shift in normal hearing and in tinnitus are not presently known. In a further analysis of results from normal hearing participants, Gander et al. (2010b, Experiment 1) applied inverse modeling to the data of each subject in order to evaluate effects of attention and training on ASSR phase and amplitude in each hemisphere separately. ASSR amplitude was larger in the right than the left hemisphere in agreement with earlier reports (Ross, 2008), and increased with attention in both hemispheres but more so in the right hemisphere. In contrast, ASSR phase changed in unison in both hemispheres and did not differ between the hemispheres. ASSR phase was not affected by attention, but shifted toward the stimulus waveform with training while ASSR amplitude remained unchanged, underscoring the independence of the response attributes. These findings suggest that interhemispheric interactions between amplitude and phase are not likely to have contributed to changes in the responses with attention or auditory experience, although such analyses have yet to be carried out on the present data.
Changes in ASSR phase and amplitude within trials are also relevant to understanding experience-induced effects. ASSR phase is a circular variable, but the nature of the phase changes (whether large or small delays or advances) can be disambiguated by referencing the ASSR response in the time domain to the onset of sound. Within trial analyses by Gander et al. (2010b) showed that during the $976 \mathrm{~ms}$ of sound stimulation the ASSR waveform shifted gradually away from the time of stimulus onset toward the zero-crossing in the stimulus waveform, stabilizing with respect to the zero-crossing after about $300 \mathrm{~ms}$ in broad agreement with fine grained analyses reported by Ross et al. (2002). Auditory training added a further shift toward the zero-crossing as the response waveform moved closer to the stimulus waveform over sessions. The training effect on ASSR phase (a latency increase with respect to sound onset) was fully expressed over the initial $488 \mathrm{~ms}$ of the stimulus which was free of target events and persisted without diminution until stimulus offset. The latter result suggests that changes in ASSR phase resulting from auditory experience were not affected by resets of the ASSR that can be evoked when salient stimuli (for example, an unexpected change in carrier frequency or a noise burst) are delivered during auditory tasks (Rohrbaugh et al., 1990a; Ross et al., 2005; Bosnyak et al., 2007). We have not observed reset responses to target stimuli in our studies, likely because the target stimuli were close to the $\mathrm{TH}$ of detection and did not alter the AM rhythm or its carrier frequency. It should be noted that reset responses shift the ASSR waveform back toward stimulus onset (a latency decrease; Ross et al., 2005) which is opposite the phase change observed during auditory training. Reset responses are also larger when behavioral responding is required (Rohrbaugh et al., 1989), vanish as the $\mathrm{TH}$ of detection is approached (Ross, 2008), and have been associated with behavioral orienting or similar change detection processes (Rohrbaugh et al., 1990b; Ross et al., 2005). These findings suggest that experience-induced phase shifts and reset responses may reflect different underlying mechanisms. However, Rohrbaugh et al. (1990b) found that reset responses showed adaptation when measured in a second session. If the processes that underlie development of the ASSR after reset are the same as those responsible for its development with sound onset (Ross et al., 2005), reset responses and phase shifts with auditory training could reflect one and the same mechanism. Additional experiments will be required to uncover the mechanisms responsible for changes in ASSR phase induced by auditory experience and to explore the neural basis of such effects.

A further gap in our knowledge concerns the role of attention in modulating auditory plasticity in humans. Because our procedures involved active performance, we have for convenience described them as training procedures. However, it has shown that passive exposure to the $40-\mathrm{Hz}$ AM training sound without task knowledge is by itself sufficient to change ASSR phase (Gander et al., 2010b, Experiment 1). Remodeling by passive exposure has also been reported for P2 amplitude by Sheehan et al. (2005), Ross and Tremblay (2009), and Gander et al. (2010b), while Tremblay et al. (2010) observed differential effects of attended performance on P2 amplitude recorded over the vertex compared to temporal-parietal regions. In our studies ASSR phase changes over sessions have tended to be greater on active 
blocks than on passive blocks, although interactions with block have not reached significance (Gander et al., 2010b). When collapsed over experiments (Bosnyak et al., 2004; Gander et al., 2010a,b, and the present findings) our results suggest that a stimulus exposure totaling $40 \mathrm{~min}$ (however, distributed in time) may be sufficient to yield a phase shift of up to $18^{\circ}$ (depending on carrier frequency) in individuals without tinnitus, that persists up to six weeks without intervening experience. This raises the possibility that procedures more efficient than the current ones (which descended from animal studies of auditory neural plasticity) could be devised to investigate effects of attention, mechanisms of ASSR phase shifts, and how experience-induced changes in ASSR phase are modified by neural changes related to tinnitus.

Looking ahead, the present findings offer only a first glimpse into how neural changes in tinnitus alter the expression of neural plasticity in auditory pathways. It is well established that tinnitus spectra (Noreña et al., 2002; Roberts et al., 2008) and residual inhibition functions (Roberts et al., 2008) overlap the region of auditory TH shift. These results have been interpreted to suggest that what neurons in the tinnitus frequency region do generates tinnitus, and stopping what they do suppresses it (Roberts et al., 2010). This line of reasoning suggests that shifts in ASSR phase may remodel normally in individuals with tinnitus, if training is given for sounds below the tinnitus frequency region. Were this result to be obtained, additional evidence of an interaction of auditory training with frequency-specific activity in tinnitus expressed in A1 would be revealed. It is also of interest to determine how ASSR amplitude evoked by probes presented to the tinnitus frequency region is affected by training below the tinnitus spectrum, and how tinnitus percepts are modulated by such training. Training with multiple frequencies outside of the tinnitus frequency region has been reported to reverse tonotopic map distortion and reduce behavioral evidence of tinnitus in rats subjected to noise trauma (Engineer et al., 2011). Similarly, passive exposure to background sound sparing the tinnitus frequency region has been reported to reduce tinnitus in humans (Okamoto et al., 2010; Teismann et al., 2011), putatively by distributing lateral inhibition to the tinnitus generating neurons. However, as noted above, passive exposure to background sound covering the tinnitus frequency/hearing loss region for weeks to months has been also reported to reduce tinnitus in humans (Davis et al., 2008) and to rescale loudness growth in individuals experiencing hyperacusis (Noreña and Chery-Croze, 2007).

\section{MATERIALS AND METHODS PARTICIPANTS}

Subjects with tinnitus $(n=12)$ were recruited by advertisements in the local newspaper, from the university ENT clinic, and our laboratory archive. Control participants $(n=12)$ were recruited from family and friends of the tinnitus subjects or from the local community. Controls reported no history of tinnitus or ear diseases. No subjects were receiving medication at the time of the study. Informed consent was obtained by procedures consistent with the Declaration of Helsinki and approved by the Research Ethics Board of McMaster University. One control participant withdrew after two sessions of auditory training owing to the occurrence of an unrelated medical illness. One tinnitus participant withdrew after the first auditory training session expressing concern that training might worsen his tinnitus. The remaining 22 participants completed training without incident. Participants were reimbursed for their parking fees and received an honorarium of $\$ 160$ for completing the study.

\section{DESIGN AND PROCEDURE}

\section{Intake session}

Participants in the tinnitus group completed a structured interview which collected detailed information on the nature and personal history of their tinnitus. The THQ was administered to assess with a standardized procedure the impact of tinnitus on quality of life (Kuk et al., 1990). Audiological measurements included an otoscopic examination, compliance testing, and measurement of THs to the limit of hearing using a GSI 61 audiometer with Telephonics 296D200 (0.125-8.0 kHz) and Sennheiser HDA $200(8.0-16 \mathrm{kHz})$ headphones (pulsed-tone method). Psychoacoustic properties of tinnitus were assessed by self-directed, computer-based tools (Roberts et al., 2008) that reported the ear of the tinnitus, its bandwidth (tonal, ringing, or hissing), loudness match for 11 sounds between 0.5 and $12.0 \mathrm{kHz}$, tinnitus frequency spectrum (likeness rating) over this same range, and a brief test for residual inhibition. Control participants completed the same intake procedure given to tinnitus subjects except for the omission of items pertaining to tinnitus.

\section{Follow-up session}

Tinnitus subjects returned one week after their last auditory training session for follow-up tinnitus measurements. The procedure was identical to their intake session except the intake questionnaire and audiological tests (audiogram, compliance) were omitted. Five tinnitus subjects did not complete the THQ in the follow-up session (the other measurements were completed) but provided their scores by mail afterwards.

\section{AUDITORY STIMULUS}

The stimulus for auditory training was a $5 \mathrm{kHz}$ pure tone AM with a $40.96 \mathrm{~Hz}$ sinusoid (called $40 \mathrm{~Hz}$ above, $100 \%$ modulation depth following the modulation wave). Stimulus duration was $976.56 \mathrm{~ms}$, allowing 40 AM pulses per stimulus (each stimulus constituting a "trial"). To determine sound level, each subject matched the loudness of the $5 \mathrm{kHz}$ training stimulus to that of a $2 \mathrm{kHz} 40-\mathrm{Hz}$ AM reference tone presented at $65 \mathrm{~dB}$ SPL. The reference tone was identical to the training stimulus used in earlier research on normal hearing subjects (Gander et al., 2010b) permitting comparison of results across studies with sound level controlled. Matching also controlled for the possibility of abnormal loudness growth (recruitment) above $2 \mathrm{kHz}$ where for some subjects in the tinnitus and control groups a degree of TH shift was present (Table 1 and Figure 2A). Stimuli were generated by a digital signal processor (Tucker-Davis RP2.1) and presented binaurally via ear inserts (Etymotic Research ER-2).

\section{TRAINING PROCEDURE}

During auditory training participants sat in a chair placed $1.4 \mathrm{~m}$ in front of a computer monitor in a sound attenuated (ambient noise level $16 \mathrm{dBA}$ SPL), electrically shielded booth. Auditory 
stimuli were presented successively separated by an ITI in which behavioral responses were recorded (Figure 1A). Approximately $2 / 3$ of the stimuli (determined randomly) contained a single amplitude-enhanced $40-\mathrm{Hz}$ pulse occurring randomly at $415 \mathrm{~ms}$, $610 \mathrm{~ms}$, or $805 \mathrm{~ms}$ after stimulus onset (target). On active blocks (Figure 1B) the word "Listen" appeared in a text box on the computer screen, instructing the participant to listen for a target event. At stimulus offset the word "Listen" on the computer screen was replaced by text asking "Did you hear a target?" Instructions beneath this text instructed the participant to press a right mouse button if a target had occurred ("yes") and a left mouse button if one had not ("no"). If the response was correct (hit or correct rejection), the text box turned green for $400 \mathrm{~ms}$ (feedback cue); if the response was incorrect (miss or false alarm) the text box turned red for $400 \mathrm{~ms}$. A variable ITI between 1400 and $1600 \mathrm{~ms}$ commenced with each behavioral response, giving an average ITI (including the feedback cue) of about $1900 \mathrm{~ms}$ depending on behavioral response latency. The computer screen remained blank after the feedback cue and changed to read "Listen" when the next trial commenced.

EEG was recorded on the first, fourth, and seventh session of auditory training, always on the same week day and time of day for each subject. In each of these sessions participants received 20 blocks of trials, each block about $2.5 \mathrm{~min}$ long and containing 54 stimuli (see Figure 1B). On alternate blocks participants performed the training task described above (active blocks) or were instructed by text on the computer screen ("Stop responding and ignore stimulus") to cease attending and wait until the next training block (passive blocks). Each session began with an active block. Sessions without EEG contained the same total number of trials as sessions with EEG except that all blocks were now active blocks.

Target events varied in the magnitude of their amplitude increase bracketing the $\mathrm{TH}$ of detection, so that a psychophysical function could be determined. Immediately prior to the first training session, a staircase procedure consisting of 80 successively presented stimuli (commencing with a $200 \%$ amplitude enhancement known to be detectable by inexperienced subjects) was used to measure the target detection $\mathrm{TH}$ for each participant. This TH was used to generate an individual stimulus set $\mathrm{TH}, \mathrm{TH}$ $\pm 5 \%, \mathrm{TH} \pm 10 \%$, and $\mathrm{TH}+20 \%$ ) suitable for learning. During training an adaptive procedure was followed in which if two targets were detected more than $95 \%$ of the time, $\mathrm{TH}$ was reduced by $5 \%$ and the remaining targets adjusted accordingly. On the last day of training the target values bracketing the detection TH measured at their amplitude peak averaged 1.57, 1.90, 2.25, 2.58, 2.90, and $3.51 \mathrm{~dB}$ above the prevailing steady-state signal. The first two of these targets were below TH and usually not detectable.

In the tinnitus group, the participant's awareness of tinnitus and its perceived loudness were probed immediately before, at the midpoint, and immediately after each training session (see Figure 1B). For each measurement, two questions appeared successively on the computer screen, each positioned above a horizontally oriented slider. The first question asked "How aware are you of your tinnitus at this moment?", and the second "How loud is your tinnitus at this moment?" Using a Powermate Controller (Griffin Technologies), subjects moved the slider to give their rating on a Borg CR100 scale with values ranging from 0 to 100 (Borg and Borg, 2001). On this scale a rating of 5 corresponded to "Almost Unaware" or "Extremely Soft," 30 to "Moderately Unaware" or "Moderate" loudness, 50 to "Aware" or "Loud," 70 to Very Aware" or "Very Loud," and 95 to "Extremely Aware" or "Extremely Loud." Subjects practiced moving the slider without any reference to tinnitus before the training session commenced.

\section{ELECTROPHYSIOLOGICAL RECORDING}

The EEG was sampled at $2048 \mathrm{~Hz}$ (DC to $417 \mathrm{~Hz}$ ) using a 128-channel Biosemi ActiveTwo amplifier (Cortech Solutions, Wilmington, NC). The electrode array was digitized for each participant (Polhemus Fastrak) prior to recording. EEG data were stored as continuous data files referenced to the vertex electrode.

\section{SIGNAL PROCESSING OF EEG DATA}

Eye blink artifacts were removed from the raw continuous data files by the spatial filtering option of BESA (version 5.1.8, MEGIS Software GmbH, Gräfelfing, Germany). EEG responses (128 channels) were then epoched including $200 \mathrm{~ms}$ pre- and poststimulus baselines.

\section{Transient responses}

EEG responses for $\sim 75 \%$ of trials (rejecting trials with surviving artifacts $>150 \mu \mathrm{V}$ ) were used for analysis of transient responses. The data were averaged and interpolated to the 81-channel "reference free" average reference montage of BESA using each participant's digitized electrode array. Subsequent filtering $(0.2-20 \mathrm{~Hz}$, zero phase) extracted P1, N1, P2, and N2 transient responses and the auditory SR. Responses were measured at electrode $\mathrm{Fz}$ where they reached their amplitude maxima. Peak amplitude and the corresponding latency were recorded for the latency windows 30$85 \mathrm{~ms}$ (P1), 85-140 ms (N1), 140-230 ms (P2), and 250-350 ms (N2).

\section{0-Hz steady-state response}

EEG responses for $\sim 90 \%$ of trials (rejecting trials with amplitude changes $>100 \mu \mathrm{V}$ ) were averaged for analysis of the ASSR, and filtered $40-42 \mathrm{~Hz}$ (zero phase) after conversion to average reference. The scalp topography of the ASSR and a digitized 128 electrode array are shown in Figure 1C (left panel) for a representative participant. The 128-channel data for each participant for the interval 244-952 ms were collapsed into a two-pulse wide waveform (Figure 1C, middle panel) and ASSR amplitude and phase determined at $40 \mathrm{~Hz}$ for each electrode by FFT (Figure 1C, right panel). Collapsing enhanced the signal-to-noise ratio and was adopted here and in previous research because changes in ASSR changes attributable to training or attention were found to be expressed throughout the stimulus interval (Gander et al., 2010b). ASSR amplitude was calculated as the total field power at $40 \mathrm{~Hz}$ summed over 128 electrodes. For calculation of ASSR phase a search algorithm, moving in steps of $0.5^{\circ}$, found the minimum angle width, encompassing electrodes on both sides of the dipolar field pattern, comprising $50 \%$ of the total $40 \mathrm{~Hz}$ power across the array. The value (in degrees) in the middle of that width 
was taken as the phase of the ASSR (Figure 1C). In infrequent cases where the absolute phase change between conditions was greater than $\pi$, phase was unwrapped by adding or subtracting $2 \pi$ to minimize the phase difference. Phase determined by this method was very close to lines determined by spatial principal component analysis but was not influenced by noisy electrodes and spurious data that do not represent the ASSR. These methods of analysis had the advantage of using all of the unmodeled data available from each participant. In previous research we found that results obtained with these methods concurred with those obtained when ASSR amplitude and phase were analyzed at electrode $\mathrm{Fz}$ where the ASSR typically reaches its amplitude maximum (Gander et al., 2010a,b). The results also concurred with those obtained by inverse modeling of the ASSR field patterns (Gander et al., 2010a,b).

Because P2 amplitude was negative for some subjects reflecting their preceding polarity-opposite $\mathrm{N} 1$ response, $\mathrm{P} 2$ data were linear transformed by adding 1.9 to each data point prior to normalization, to remove negative values and prevent division by zero (after Gander et al., 2010a).

\section{STATISTICAL EVALUATION}

Repeated measures ANOVAS were performed using the General Linear Model of Statistica (version 6.0). Repeated measures having more than two levels were Greenhouse-Geisser corrected. Unless stated otherwise, significance level was set at $\alpha=0.05$ (two-tailed). Least significant difference (LSD) tests were used to describe significant main effects and interactions.

\section{Behavioral data}

Behavioral performance was evaluated for each participant by calculating the mean probability of a hit $[P(\mathrm{Hit})$, a target reported when one was present] collapsed over target amplitudes and sessions, and the mean probability of a false alarm $[P(\mathrm{FA})$, a target reported when target amplitude was zero] collapsed over sessions. The difference between these probabilities was used to assess group performance. A psychophysical function was also constructed for each participant and session by plotting $P$ (Hit) as a function of target amplitude and fitting a logistic $\left[f_{(x)}=0.5 /\{1+\right.$ $\exp [-$ slope $\times($ amp increase - threshold $)]\}+0.5]$ using a maximum likelihood method with the Palamedes psychophysics toolbox for Matlab (Prins and Kingdom, 2009). Changes in TH (the amplitude increase corresponding to $75 \%$ correct) over sessions were assessed for the tinnitus and control groups separately. These changes were modeled for each participant assuming that the slopes followed an exponential decay but allowing bidirectional

\section{REFERENCES}

Alain, C., Snyder, J. S., He, Y., and Reinke, K. S. (2007). Changes in auditory cortex parallel rapid perceptual learning. Cereb. Cortex 17, 1074-1084.

Borg, G., and Borg, E. (2001). A new generation of scaling methods: level anchored ratio scaling. Psychologica 28, 15-45.

Baynton, S. (2010). The Development of Neural Plasticity in the Human
Auditory System. Unpublished MSc thesis (preview available at www. hnplab.mcmaster.ca).

Bosnyak, D. J., Eaton, R. A., and Roberts, L. E. (2004). Distributed auditory cortical representations are trained at pitch discrimination with $40 \mathrm{~Hz}$ amplitude modulated tones. Cereb. Cortex 14, 1088-1099.

Bosnyak, D. J., Stevens, S., Gander, P. E., and Roberts, L. E. (2007). "Phase modified when non-musicians are

changes such that the results were driven by the data (Prins and Kingdom, 2009).

It should be noted that the $\mathrm{TH}$ for target detection determined prior to training by the staircase procedure that was administered to each participant gave values (mean $\mathrm{TH}=68 \%$ for tinnitus, $74 \%$ for controls, difference not significant) that were high compared to earlier experiments where young normal hearing subjects were trained with a $2 \mathrm{kHz} 40-\mathrm{Hz}$ AM sound (mean $\mathrm{TH}=31 \%$, Gander et al., 2010b). The reason for this difference may relate to the carrier frequency used in the present study and the presence of some degree of hearing loss at $5 \mathrm{kHz}$ in tinnitus and control participants (Table 1). However, in the first training session most (but not all) of the targets calculated from each participant's TH quickly became detectable, giving THs determined from individual psychophysical functions $(\mathrm{TH}=35.1 \%$ on day 1 of Figure 3B) that were closer to previous results for normal hearing subjects. Changes in THs over sessions gave a coherent picture of discrimination performance during training, but changes in slope did not, because for many subjects slopes were uncommonly steep in the initial training sessions. Therefore, we relied on $\mathrm{TH}$ changes to depict changes in performance over sessions (Figure 3B). It was also possible to construct a psychophysical function from the collapsed data of each group on sessions (Figure 3A).

\section{EEG data}

Because individual differences in ASSR amplitude are large, likely reflecting stable anatomical differences among subjects in the orientation and strength of ASSR generators and their summation across tonotopic maps in Heschl's gyrus, ASSR amplitude for each subject was normalized by dividing each participant's data by the mean of the passive block of their respective group on day 1 (after Gander et al., 2010b). This step referenced the effects of training and task to a common passive baseline within each group. For purposes of comparison ASSR phase and P2 amplitude were normalized by the same method, although these measures are typically less variable between-subjects than ASSR amplitude.

\section{ACKNOWLEDGMENTS}

Support from a grant by the Tinnitus Research Initiative is gratefully acknowledged. Additional funding was provided by the Natural Sciences and Engineering Research Council of Canada. We thank Victoria Mosher for assistance and our participants for undertaking the study.

dynamics in the $40 \mathrm{hz}$ auditory steady state response," in New Frontiers in Biomagnetism, eds D. Cheyne, B. Ross, G. Stroink, and H. Weinberg, International Congress Series (Amsterdam: Elsevier), 29-32.

Brugge, J. F., Nourski, K. V., Oya, H., Reale, R. A., Kawasaki, H., Steinschneider, M., and Howard, M. A. (2009). Coding of repetitive transients by auditory cortex on heschl's gyrus. J. Neurophysiol. 102, 2358-2374.

Carpenter, M., Cranford, J. L., Hymal, M. R., DeChicchis, A. R., and Holbert, D. (2002). Electrophysiologic signs of attention versus distraction in a binaural listening task. J. Clin. Neurophysiol. 19, 55-60.

Davis, P. B., Wilde, R. A., Steed, L. G., and Hanley, P. J. (2008). Treatment of tinnitus with a customized acoustic neural stimulus: a controlled 
clinical study. Ear Nose Throat J. 87, 330-339.

Eggermont, J. J., and Roberts, L. E. (2004). The neuroscience of tinnitus. Trends Neurosci. 27, 676-682.

Engineer, N. D., Riley, J. R., Seale, J. D., Vrana, W. A., Shetake, J. A., Sudanagunta, S. P., Borland, M. S., and Kilgard, M. P. (2011). Reversing pathological neural activity using targeted plasticity. Nature 470, 101-104.

Finlayson, P. G., and Kaltenbach, J. A. (2009). Alterations in the spontaneous discharge patterns of single units in the dorsal cochlear nucleus following intense sound exposure. Hear. Res. 256, 104-117.

Formby, C., Sherlock, L. P., and Gold, S. L. (2003). Adaptive plasticity of loudness induced by chronic attenuation and enhancement of the acoustic background. J. Acoust. Soc. Am. 114, 55-58.

Formisano, E., Kim, D. S., Di Salle, F., van de Moortele, P. F., Ugurbil, K., and Goebel, R. (2003). Mirrorsymmetric tonotopic maps in human primary auditory cortex. Neuron 40, 859-869.

Gander, P. E., Bosnyak, D. J., and Roberts, L. E. (2010a). Evidence for modality-specific but not frequency-specific modulation of human primary auditory cortex by attention. Hear. Res. 268, 213-226.

Gander, P. E., Bosnyak, D. J., and Roberts, L. E. (2010b). Acoustic experience but not attention modifies neural population phase expressed in human primary auditory cortex. Hear. Res. 269, 81-94.

Hillyard, S. A., Hink, R. F., Schwent, V. L., and Picton, T. W. (1973). Electrical signs of selective attention in the human brain. Science 182, 177-180.

Henry, J. A., and Meikle, M. B. (2000). Psychoacoustic measures of tinnitus. J. Am. Acad. Audiol. 11, 138-155.

Hoare, D. J., Kowalkowski, V. L., Kang, S., and Hall, D. A. (2011). Systematic review and metaanalyses of randomized controlled trials examining tinnitus management. Laryngoscope 121, 1555-1564.

Kaltenbach, J. A., Zacharek, M. A., Zhang, J., and Frederick, S. (2004). Activity in the dorsal cochlear nucleus of hamsters previously tested for tinnitus following intense tone exposure. Neurosci. Lett. 355, 121-125.

Kilgard, M. P., Pandya, P. K., Vazquez, J., Gehi, A., Schreiner, C. E., and Merzenich, M. M. (2001). Sensory input directs spatial and temporal plasticity in primary auditory cortex. J. Neurophysiol. 86, 326-338.

Kaas, J. H., and Hackett, T. A. (2000). Subdivisions of auditory cortex and processing streams in primates. Proc. Natl. Acad. Sci. U.S.A. 97, 11793-11799.

Kiss, J., and Patel, A. J. (1992). Development of the cholinergic fibres innervating the cerebral cortex of the rat. Int. J. Dev. Neurosci $10,153-170$.

Kujawa, S. G., and Liberman, M. C. (2009). Adding insult to injury: cochlear nerve degeneration after "temporary" noise-induced hearing loss. J. Neurosci. 29, 14077-14085.

Kuk, F. K., Tyler, R. S., Russell, D., and Jordan, H. (1990). The psychometric properties of a tinnitus handicap questionnaire. Ear Hear. 11, 434-445.

Metherate, R., and Ashe, J. H. (1993). Nucleus basalis stimulation facilitates thalamocortical synaptic transmission in the rat auditory cortex. Synapse 14, 132-143.

Noreña, A. J. (2011). An integrative model of tinnitus based on a central gain controlling neural sensitivity. Neurosci. Biobehav. Rev. 35, 1089-1090.

Noreña., A. J., and Chery-Croze, S. (2007). Enriched acoustic environment rescales auditory sensitivity. Neuroreport 18, 1251-1255.

Noreña, A. J., and Eggermont, J. J. (2003). Changes in spontaneous neural activity immediately after an acoustic trauma: implications for neural correlates of tinnitus. Hear. Res. 183, 137-153.

Noreña, A., and Eggermont, J. J. (2005). Enriched acoustic environment after noise trauma reduces hearing loss and prevents cortical map reorganization. J. Neurosci. 25 , 699-705.

Noreña, A., Micheyl, C., ChéryCroze, S., and Collet, L. (2002). Psychoacoustic characterization of the tinnitus spectrum: implications for the underlying mechanisms of tinnitus. Audiol. Neurootol. 7, 358-369.

Noreña, A. J., Tomita, M., and Eggermont, J. J. (2003). Neural changes in cat auditory cortex after a transient pure-tone trauma. J. Neurophysiol. 90, 2387-2401.

Okamoto, H., Stracke, H., Stoll, W., and Pantev, C. (2010). Listening to tailor-made notched music reduces tinnitus loudness and tinnitusrelated auditory cortex activity.
Proc. Natl. Acad. Sci. U.S.A. 107, 1207-1210

Picton, T. W., Alain, C., Woods, D. L., John, M. S., Scherg, M., Valdes-Sosa, P., Bosch-Bayard, J., and Trujillo, N. J. (1999). Intracerebral sources of human auditory-evoked potentials. Audiol. Neurootol. 4, 64-79.

Pienkowski, M., and Eggermont, J. J. (2010). Passive exposure of adult cats to moderate-level tone pip ensembles differentially decreases AI and AII responsiveness in the exposure frequency range. Hear Res. 268, 151-162.

Pozo, K., and Goda, Y. (2010). Unraveling mechanisms of homeostatic synaptic plasticity. Neuron 66 , 337-351.

Prins, N., and Kingdom, F. A. A. (2009). Palamedes: matlab routines for analyzing psychophysical data. Available online at http:// www.palamedestoolbox.org

Rajan, R., and Irvine, D. R. F. (1998) Neuronal responses across cortical field A1 in plasticity induced by peripheral auditory organ damage. Audiol. Neurootol. 3, 123-144.

Reinke, K. S., He, Y., Wang, C., and Alain, C. (2003). Perceptual learning modulates sensory evoked response during vowel segregation. Cogn. Brain Res. 17, 781-791.

Roberts, L. E., and Bosnyak, D. J. (2010a). "Auditory training in tinnitus," in The Textbook of Tinnitus, eds A. Møller, T. Kleinjung, B. Langguth, and D. de Ridder (New York, NY: Springer), 563-574.

Roberts, L. E., and Bosnyak, D. J. (2010b). Evidence for depression of auditory cortical synapses in tinnitus and its reversal in residual inhibition. Soc. Neurosci. 170.22.

Roberts, L. E., Eggermont, J. J., Caspary, D. C., Shore, S. E., Melcher, J. R., and Kaltenbach, J. A. (2010). Ringing ears: the neuroscience of tinnitus. J. Neurosci. 30, 14980-14986.

Roberts, L. E., Moffat, G., Baumann, M., Ward, L. M., and Bosnyak, D. J. (2008). Residual inhibition functions overlap tinnitus spectra and the region of auditory threshold shift. J. Assoc. Res. Otolaryngol. 9, 417-435.

Rohrbaugh, J. W., Varner, J. L., Paige, S. R., Eckardt, M. J., and Ellingson, R. J. (1989). Event-related perturbations in an electrophysiological measure of auditory function: a measure of sensitivity during orienting? Biol. Psychol. 29, 247-271.

Rohrbaugh, J. W., Varner, J. L., Paige, S. R., Eckardt, M. J., and Ellingson, R. J. (1990a). Auditory and visual event-related perturbations in the $40 \mathrm{~Hz}$ auditory steady-state response. Electroencephaloqr. Clin. Neurophysiol. 76, 148-164.

Rohrbaugh, J. W., Varner, J. L., Paige, S. R., Eckardt, M. J., and Ellingson, R. J. (1990b). Event-related perturbations in an electrophysiological measure of auditory sensitivity: effects of probability, intensity and repeated sessions. Int. J. Psychophysiol. 10, 17-32.

Ross, B. (2008). A novel type of auditory responses: temporal dynamics of $40-\mathrm{Hz}$ steady-state responses induced by changes in sound localization. J. Neurophysiol. 100, 1265-1277.

Ross, B., Herdman, A. T., and Pantev, C. (2005). Stimulus induced desynchronization of human auditory $40-\mathrm{Hz}$ steady-state responses. J. Neurophysiol. 94, 4082-4093.

Ross, B., Picton, T. W., Herdman, A. T., and Pantev, C. (2004). The effect of attention on the auditory steady-state response. Neurol. Clin. Neurophysiol. 22. November 30.

Ross, B., Picton, T. W., and Pantev, C. (2002). Temporal integration in the human auditory cortex as represented by the development of the steady-state magnetic field. Hear. Res. 165, 68-84.

Ross, B., and Tremblay, K. (2009). Stimulus experience modifies auditory neuromagnetic responses in young and older listeners. Hear. Res. 248, 48-59.

Sarter, M., Hasselmo, M. E., Bruno, J. P., and Givens, B. (2005). Unraveling the attentional functions of cortical cholinergic inputs: interactions between signal-driven and cognitive modulation of signal detection. Brain Res. Rev. 48, 98-111.

Schaette, R., and McAlpine, D. (2011). Tinnitus with a normal audiogram: physiological evidence for hidden hearing loss and computational model. J. Neurosci. 31, 13452-13457.

Scholl, B., and Wehr, M. (2008). Disruption of balanced cortical excitation and inhibition by acoustic trauma. J. Neurophysiol. 100, 646-656.

Seki, S., and Eggermont, J. J. (2003). Changes in spontaneous firing rate and neural synchrony in cat primary auditory cortex after localized toneinduced hearing loss. Hear. Res. 180, 28-38.

Sereda, M., Hall, D. A., Bosnyak, D. J., Edmondson-Jones, M., Roberts, L. E., Adjamian, P., and Palmer, A. R. (2011). Re-examining the relationship between audiometric profile and tinnitus pitch. Int. J. Audiol. $50,303-312$. 
Sheehan, K. A., McArthur, G. M., and Bishop, D. V. (2005). Is discrimination training necessary to cause changes in the P2 auditory event-related brain potential to speech sounds? Cogn. Brain Res. 25, 547-553.

Song, J. H., Skoe, E., Wong, P. C. M., and Kraus, N. (2008). Plasticity in the adult human auditory brainstem following short-term linguistic learning. J. Cogn. Neurosci. 20, 1892-1902.

Teismann, H., Okamoto, H., and Pantev, C. (2011). Short and intense tailor-made notched music training against tinnitus: the tinnitus frequency matters. PLoS ONE 6:e24685. doi: 10.1371/journal.pone.0024685

Tremblay, K., Kraus, N., McGee, T., Ponton, C., and Otis, B. (2001). Central auditory plasticity: changes in the N1-P2 complex after speechsound training. Ear Hear. 22, 79-90.

Tremblay, K. L., Inoue, K., McClannahan, K., and Ross, B. (2010). Repeated stimulus exposure alters the way sound is encoded in the human brain. PLOS ONE 5:e10283. doi: 10.1371/journal. pone. 0010283

Turrigiano, G. G., and Nelson, S. B. (2004). Homeostatic plasticity in the developing nervous system. Nat. Rev. Neurosci. 5, 97-107.

Tzounopoulos, T., and Kraus, N (2009). Learning to encode timing: mechanisms of plasticity in the auditory brainstem. Neuron 62, 463-469.

Wang, H. N., Brozoski, T. J., Turner, J. G., Ling, L. L., Parrish, J. L., Hughes, L. F., and Caspary, D. M. (2009). Plasticity at glycinergic synapses in the dorsal cochlear nucleus of rats with behavioral evidence of tinnitus. Neuroscience 164, 747-759.

Weisz, N., Hartmann, T., Dohrmann, K., Schlee, W., and Noreña, A. (2006). High-frequency tinnitus without hearing loss does not mean absence of deafferentation. Hear. Res. 222, 108-114.
Weisz, N., Müller, S., Schlee, W., Dohrmann, K., Hartmann, T., and Elbert, T. (2007). The neural code of auditory phantom perception. J. Neurosci. 26, 1479-1484.

Wienbruch, C., Paul, I., Weisz, N., Elbert, T., and Roberts, L. E. (2006). Frequency organization of the $40-\mathrm{Hz}$ auditory steady-state response in normal hearing and in tinnitus. Neuroimage 33, 180-194.

Yao, H., and Dan, Y. (2001). Stimulus timing-dependent plasticity in cortical processing of orientation. Neuron 32, 315-323.

Zeng, C., Nannapaneni, N., Zhou, J., Hughes, L. F., and Shore, S. (2009). Cochlear damage changes the distribution of vesicular glutamate transporters associated with auditory and nonauditory inputs to the cochlear nucleus. J. Neurosci. 29, 4210-4217.

Zhou, X., Henin, S., Long, G. R. and Parra, L. C. (2011). Impaired cochlear function correlates with the presence of tinnitus and its estimated spectral profile. Hear. Res. 277, 107-116.

Conflict of Interest Statement: The authors declare that the research was conducted in the absence of any commercial or financial relationships that could be construed as a potential conflict of interest.

Received: 14 February 2012; accepted: 05 May 2012; published online: 28 May 2012.

Citation: Roberts LE, Bosnyak DJ and Thompson DC (2012) Neural plasticity expressed in central auditory structures with and without tinnitus. Front. Syst. Neurosci. 6:40. doi: 10.3389/fnsys. 2012.00040

Copyright (c) 2012 Roberts, Bosnyak and Thompson. This is an open-access article distributed under the terms of the Creative Commons Attribution Non Commercial License, which permits noncommercial use, distribution, and reproduction in other forums, provided the original authors and source are credited. 